\title{
REVIEWS
}

\section{Immunity against Staphylococcus aureus cutaneous infections}

\author{
Lloyd S. Miller* ${ }^{* \ddagger}$ and John S. Cho* \\ Abstract | Complications arising from cutaneous and soft tissue infections with \\ Staphylococcus aureus are a major clinical problem owing to the high incidence of \\ these infections and the widespread emergence of antibiotic-resistant bacterial strains. \\ If prophylactic vaccines or immunotherapy for certain patient populations are to be \\ developed as an alternative to antibiotics, it will be essential to better understand the immune \\ mechanisms that provide protection against $S$. aureus skin infections. Recent discoveries \\ have identified a key role for interleukin-1 (IL-1)- and IL-17-mediated immune responses \\ in promoting neutrophil recruitment to the site of infection in the skin, a process that is \\ required for host defence and bacterial clearance. This Review describes these new insights \\ and discusses their potential impact on immune-based therapies and vaccination strategies.
}

Methicillin-resistant S. aureus

(MRSA). Staphylococcus aureus strains that have developed resistance to $\beta$-lactam antibiotics, such as penicillin, methicillin and cephalosporins, and are becoming increasingly resistant to other classes of antibiotics.

* Department of Medicine, Division of Dermatology. University of California Los Angeles (UCLA), 52-121 Center for Health Sciences, 10833 Le Conte Avenue, Los Angeles, California 90095, USA.

${ }^{\ddagger}$ Department of Orthopaedic

Surgery, University of California Los Angeles (UCLA), Los Angeles, California 90095, USA.

Correspondence to L.S.M. e-mail:

lloydmiller@mednet.ucla.edu doi:10.1038/nri3010

Published online 1 July 2011
Staphylococcus aureus is responsible for the majority of skin and soft tissue infections in humans, including impetigo, folliculitis, cellulitis and infected ulcers and wounds ${ }^{1,2}$. In addition, $S$. aureus can cause invasive and life-threatening infections, such as bacteraemia, abscesses, pneumonia, osteomyelitis, meningitis, endocarditis and sepsis ${ }^{3}$. Treatment of these infections has been complicated by the emergence of methicillin-resistant S. aureus (MRSA) strains, which are becoming increasingly resistant to multiple antibiotics ${ }^{4,5}$. Invasive MRSA infections result in more deaths annually $(\sim 18,500)^{6}$ than any other single infectious agent in the United States, exceeding the number of deaths associated with HIV/ AIDS, viral hepatitis and influenza combined ${ }^{7}$. MRSA infections are endemic in hospitals worldwide; in addition, community-associated MRSA (CA-MRSA) can cause infections in otherwise healthy individuals $\mathrm{s}^{4,5}$ and is responsible for a significant percentage of $S$. aureus skin and soft tissue infections in the United States $(>50 \%)^{2}$, Asian countries $(\sim 17 \%)^{8}$ and Europe (ranging from $<1 \%$ to $32 \%$, depending on the country $)^{9-11}$. USA300 has emerged as the most prevalent CA-MRSA isolate in the United States and is commonly associated with skin and soft tissue infections $s^{4,5}$. It should be mentioned that colonization of the skin and mucosa with S. aureus (and MRSA) is a risk factor for invasive infection, which is concerning as approximately one-third of the population in the United States is colonized with S. aureus ${ }^{12}$.

Uncomplicated $S$. aureus skin infections - such as impetigo, infected abrasions and folliculitis - can generally be managed in an outpatient setting with oral and topical antibiotic therapy and/or incision and drainage 4 . By contrast, complicated $S$. aureus skin infections that spread to deeper soft tissues can be particularly difficult to treat and often require hospitalization, intravenous antibiotics and sometimes surgery ${ }^{13,14}$. Such infections include cellulitis and infected leg ulcers and wounds in patients with diabetes mellitus or venous insufficiency. It is important to understand the protective immune responses against $S$. aureus infections in the skin for the future development of an effective prophylactic vaccination strategy, which would be a major therapeutic advance to prevent these infections altogether (in particular, those caused by virulent MRSA strains, such as USA300). In addition, immunomodulatory therapies could be developed for use in certain patient populations, such as patients with congenital or acquired immune defects who are at risk for $S$. aureus infections, patients with frequent recurrences of infection despite numerous decolonization attempts and patients with complicated S. aureus skin infections.

The skin is an important barrier that protects the body from pathogenic microorganisms encountered in the environment ${ }^{15,16}$. In addition, when the epidermal barrier is breached, the skin also functions as a first responder in triggering cutaneous innate and adaptive immune responses ${ }^{15,16}$. Recently, the identification of specific immune impairments in humans with genetic immunodeficiency conditions that predispose them to $S$. aureus infections has provided new insights into the key immune responses that are involved in protection against these infections. With particular relevance 
T helper 17 cell

( $T_{H} 17$ cell). A subset of $T$ cells that is characterized by the ability to produce interleukin-17 (IL-17A and IL-17F). These cells also produce IL-21, IL-22 and IL-26 and express the transcription factor retinoic acid receptorrelated orphan receptor- $\gamma \mathrm{t}$ (RORyt). $T_{H} 17$ cells have an important role in host defence against extracellular pathogens and in autoimmune diseases.

Hyper-IgE syndrome A rare hereditary disorder characterized by recurrent S. aureus infections in the skin and the lung, severe eczema-like skin rashes and high serum IgE levels. Mutations in STAT3 and DOCK8 were recently identified as causing autosomal dominant and recessive forms of this disease, respectively. The susceptibility to infections is believed to be caused by a deficiency of $T_{H} 17$ cells.

Neutrophil abscess A collection of pus that is comprised largely of dead neutrophils formed in tissues or organs in response to an infection, in particular pyogenic (pus-forming) infections such as with $S$. aureus. A neutrophil abscess is surrounded by an abscess wall or capsule that prevents the spread of infection to adjacent healthy tissue.

Antimicrobial peptides Polypeptides, typically less than 100 amino acids, that have an important role in innate immune responses by providing broad-spectrum antimicrobial activity against pathogenic microorganisms There are different types of antimicrobial peptide that promote microbial killing by various mechanisms, but many function by disrupting the membranes of microorganisms. to the skin, certain patients who suffer from recurrent and severe $S$. aureus cutaneous infections were found to have impaired interleukin-1 receptor (IL-1R) and/or Toll-like receptor (TLR) signalling (as a result of myeloid differentiation primary response protein 88 (MYD88) or IL-1R-associated kinase 4 (IRAK4) deficiency) ${ }^{17-20}$ or impaired $\mathrm{T}$ helper 17 cell $\left(\mathrm{T}_{\mathrm{H}} 17\right.$ cell) responses (as a result of hyper-IgE syndrome $)^{21-24}$. In mouse models of S. aureus cutaneous infection, IL-1-mediated ${ }^{25-27}$ and IL-17-mediated ${ }^{28,29}$ responses have further been shown to be crucial for host defence by promoting neutrophil abscess formation in the skin, which is required for bacterial clearance. These discoveries have brought IL-1 and IL-17 to the forefront of the field and indicate that immune-based strategies aimed at promoting these responses might provide a therapeutic advantage in certain patient populations.

Although certain immune mechanisms have been shown to contribute to host defence against $S$. aureus infections in the bloodstream and in various organs and tissues ${ }^{30}$, this Review focuses on immune responses against $S$. aureus infections in the skin. In addition, S. aureus and MRSA virulence factors and immune evasion mechanisms have been reviewed elsewhere e $^{5,31,32}$. This Review highlights the recent advances involving the role of IL-1- and IL-17-mediated responses in host defence against $S$. aureus cutaneous infections and discusses their implications for future immunotherapy and vaccine development.

\section{Anatomy of the skin immune system}

The skin has constitutive innate immune mechanisms that help to protect against pathogens (FIG. 1a). On the surface of the skin, the presence of commensal microorganisms that occupy niches suitable for bacterial growth combined with a low temperature and $\mathrm{pH}$ creates an environment that resists pathogen growth ${ }^{33}$. In particular, the commensal microorganisms that comprise the skin microbiome are an area of active investigation ${ }^{34}$, and the constituents of the microbiome might affect $S$. aureus colonization. The efforts to decipher the human skin microbiome are reviewed elsewhere ${ }^{33}$. The uppermost layer of the epidermis, the corneal layer, is a unique layer that is not present in other epithelia exposed to the environment (such as the gut and lung epithelia) ${ }^{15,16}$. This layer is comprised of dead keratinocytes that are devoid of organelles, and it provides the physical barrier of the skin ${ }^{15,16}$. In addition, the corneal layer contains antimicrobial peptides, which are produced by keratinocytes and can be induced in response to infection. Such peptides include human $\beta$-defensin 2 , human $\beta$-defensin 3, cathelicidins and RNase 7, which have bacteriostatic or bactericidal activity against $S$. aureus ${ }^{35,36}$. Beneath the corneal layer are the granular, spinous and basal layers. These layers are composed of keratinocytes that express pattern recognition receptors (PRRs) - such as TLRs and nucleotide-binding oligomerization domain proteins (NODs) - that sense the pathogen-associated molecular patterns (PAMPs) of invading microorganisms to initiate early cutaneous immune responses ${ }^{30,37}$. In addition to keratinocytes, other cells that reside in human skin also express PRRs and contribute to the cutaneous immune responses discussed elsewhere ${ }^{15,16}$. Such cells include Langerhans cells and $\gamma \delta$ T cells (mice only) in the epidermis and macrophages, dendritic cells, mast cells, $\mathrm{B}$ and T cells, plasma cells, natural killer (NK) cells and fibroblasts in the dermis ${ }^{15,16}$.

Neutrophil recruitment to the skin. A hallmark of S. aureus infections is neutrophil abscess formation, which is required for bacterial clearance ${ }^{38}$. The recruitment of neutrophils to a site of $S$. aureus infection in the skin involves pathogen recognition by the PRRs of the innate immune system (see above) and the subsequent initiation of proinflammatory immune responses (FIG. 1 b). Neutrophil recruitment from the circulation is mediated by multiple factors, including pro-inflammatory cytokines (such as IL- $1 \alpha$, IL-1 $\beta$, tumour necrosis factor (TNF) and IL-6) and chemokines. In particular, the chemokines CXCchemokine ligand 1 (CXCL1; also known as GRO $\alpha$ in humans and $\mathrm{KC}$ in mice), CXCL2 (also known as MIP2), CXCL5 (also known as ENA78) and CXCL8 (also known as IL-8) mediate the chemoattraction of neutrophils through CXC-chemokine receptor 2 (CXCR2), which is expressed on neutrophils. Also required are adhesion molecules that promote neutrophil rolling, adhesion and diapedesis; these molecules include P-selectin, E-selectin and intercellular adhesion molecule 1 (ICAM1) on the endothelium and L-selectin and lymphocyte functionassociated antigen 1 (LFA1; also known as $\alpha \mathrm{L} \beta 2$ integrin) on neutrophils ${ }^{39}$.

In addition, it has been shown that neutrophil abscess formation during $S$. aureus infection in mouse skin requires three mechanisms: neutrophil recruitment to the skin from the circulation; prolonged neutrophil survival in the abscess; and the homing of $\mathrm{KIT}^{+}$progenitor cells to the abscess, where they locally give rise to mature neutrophils ${ }^{40}$. Once neutrophils encounter S. aureus in the skin, they use multiple mechanisms to facilitate bacterial killing (FIC. 1 c), including phagocytosis to engulf the bacteria and oxidative burst to generate reactive oxygen species (such as superoxide $\left(\mathrm{O}_{2}^{-}\right)$, hydrogen peroxide $\left(\mathrm{H}_{2} \mathrm{O}_{2}\right)$ and hypochlorous acid $\left.(\mathrm{HOCl})\right)$ that mediate bacterial killing. They also produce antimicrobial peptides (such as cathelicidins, lysozyme and $\alpha$-defensins) that have direct microbicidal activity, and proteinases (such as cathepsin G, neutrophil elastase and proteinase 3 (also known as myeloblastin)) and acid hydrolases that degrade bacterial components. Finally, they express proteins that sequester essential nutrients to limit bacterial growth (such as lactoferrin, transcobalamin II, neutrophil gelatinase-associated lipocalin (NGAL) and calprotectin $)^{41-43}$.

$T_{H} 17$ cells and cutaneous immune responses. $\mathrm{T}_{\mathrm{H}} 17$ cells are a subset of $\mathrm{CD}^{+} \mathrm{T}_{\mathrm{H}}$ cells that produce IL-17 (both IL-17A and IL-17F), as well as IL-21, IL-22 and IL-26 (REFS 44,45). $\mathrm{T}_{\mathrm{H}} 17$ cells have an important role in immune responses at epithelial sites, including the $\operatorname{skin}^{44,45}$. IL-17A and IL-17F promote neutrophil recruitment and abscess formation by inducing the expression of neutrophil-attracting chemokines (such as CXCL1, CXCL2, CXCL5 and CXCL8) and granulopoiesis factors 
a

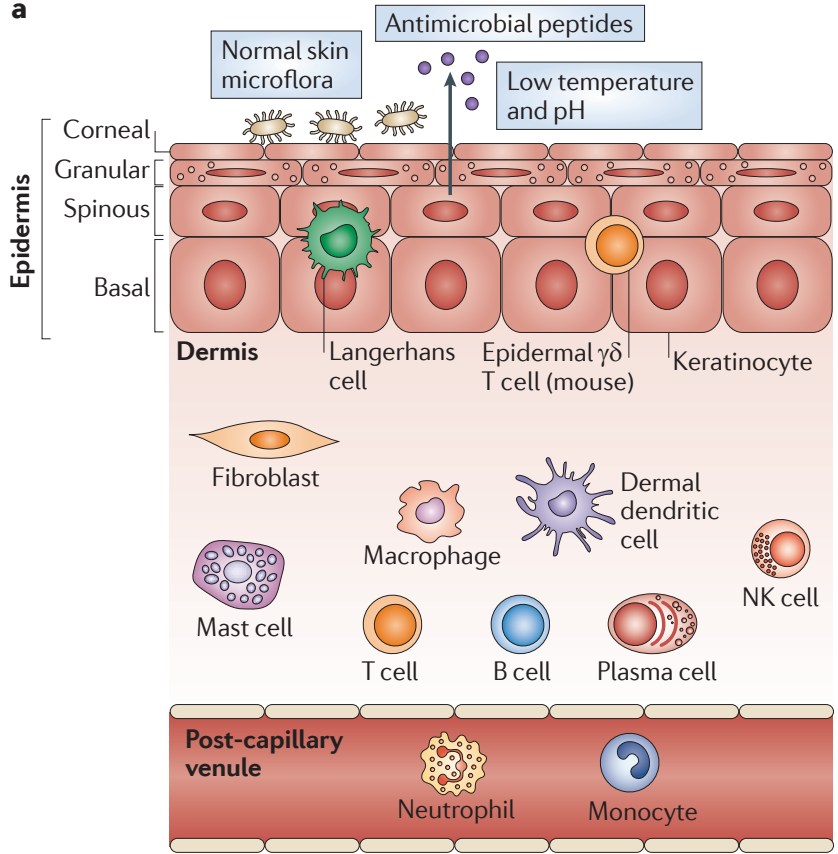

b

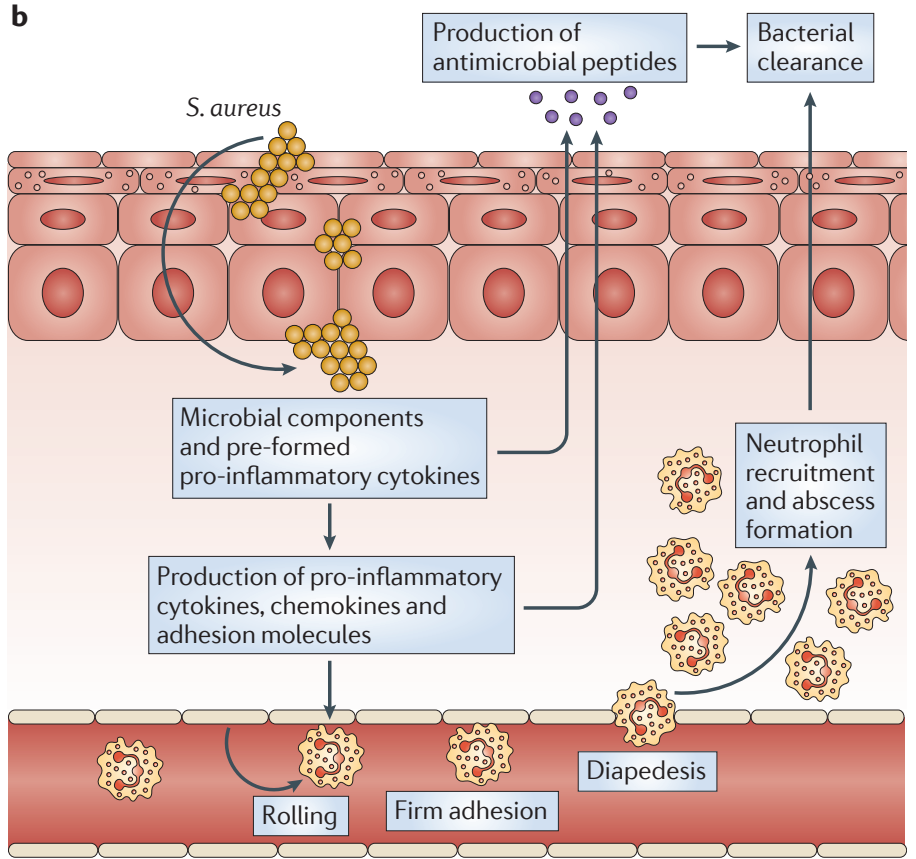

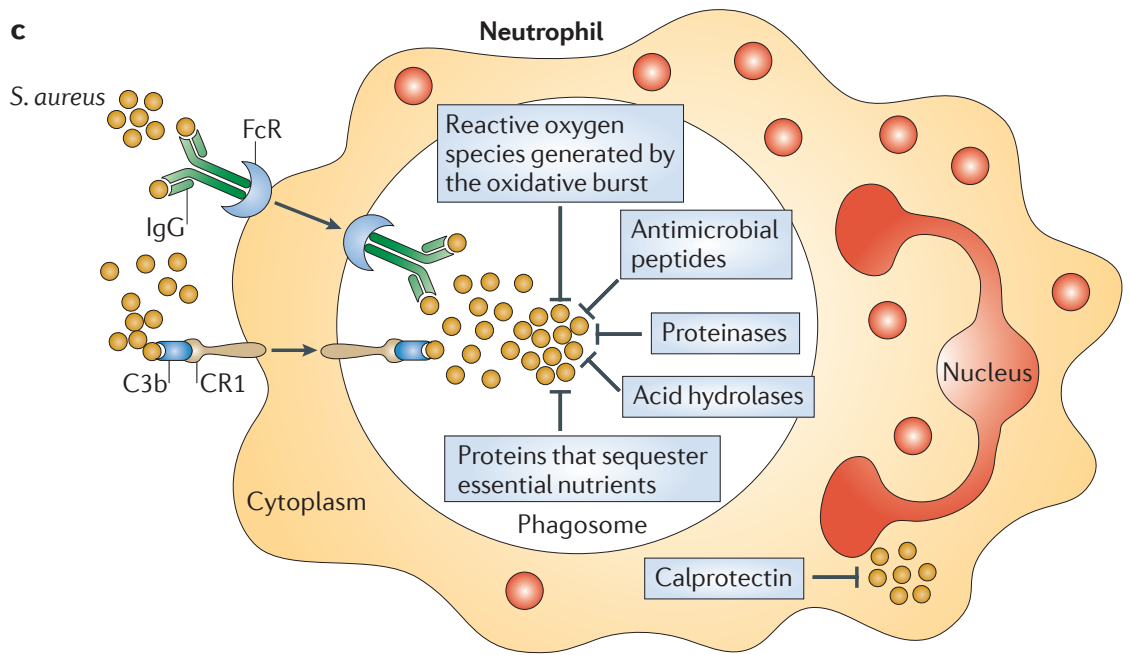

Figure 1 | Anatomy of cutaneous immune responses. a | Normal skin has constitutive innate immune mechanisms, including the epidermal barrier, normal skin microflora, antimicrobial peptides and a low temperature and pH. These mechanisms help to prevent infection by pathogenic microorganisms from the environment. $\mathbf{b} \mid$ During a Staphylococcus aureus cutaneous infection, the epidermal barrier is breached and keratinocytes and resident skin immune cells (such as Langerhans cells and $\gamma \delta$ T cells in the epidermis, and dendritic cells, macrophages, fibroblasts, mast cells, B and T cells, plasma cells and natural killer (NK) cells in the dermis) produce pro-inflammatory cytokines, chemokines and adhesion molecules. These molecules promote the recruitment from the bloodstream of neutrophils, which help to control the infection by forming an abscess. Neutrophil abscess formation is a hallmark of S. aureus infections (which are typically characterized as pyogenic infections) and is required for bacterial clearance.

Pattern recognition receptors

(PRRS). Receptors found on host cells that recognize conserved components of microorganisms (known as pathogen-associated molecular patterns) and initiate the production of pro-inflammatory immune responses. These receptors include Toll-like receptors and nucleotide-binding oligomerization domain proteins.
Pro-inflammatory cytokines also induce the production of antimicrobial peptides (such as $\beta$-defensins and cathelicidins) that have bacteriostatic or bactericidal activity against S. aureus. $\mathbf{c}$ | Neutrophils phagocytose opsonized bacteria using Fc and complement receptors. Following this uptake into phagosomes, there are multiple mechanisms that promote bacterial killing. The first is the oxidative burst, which generates reactive oxygen species (such as $\mathrm{O}_{2}^{-}, \mathrm{H}_{2} \mathrm{O}_{2}$ and $\mathrm{HOCl}$ ) through NADPH oxidase and myeloperoxidase. These species can directly kill bacteria but also produce a charge in the phagocytic membrane to promote enzymatic killing. Second, the phagosome contains antimicrobial peptides (such as cathelicidins, lysozyme, azurocidin and a-defensins), which have direct microbicidal activity. Third, proteinases (such as cathepsin G, neutrophil elastase, gelatinase, neutrophil collagenase and proteinase 3) and acid hydrolases degrade bacterial components. Last, proteins that sequester essential nutrients limit bacterial growth; such proteins include lactoferrin (which sequesters iron and copper), transcobalamin II (which binds vitamin B12) and neutrophil gelatinaseassociated lipocalin (NGAL; which binds bacterial siderophores, preventing the extraction of iron). If S. aureus enters the cytoplasm of neutrophils, there is an abundance of calprotectin, which sequesters $\mathrm{Mn}^{2+}$ and $\mathrm{Zn}^{2+}$ to inhibit bacterial growth. C3b, complement component C3b; CR1, complement receptor type 1 . 


\begin{tabular}{|c|c|}
\hline Immune defect & Diseases \\
\hline \multicolumn{2}{|l|}{ Neutrophils } \\
\hline Neutropenia & $\begin{array}{l}\text { Severe congenital neutropenia and neutropenic patients (such as patients } \\
\text { undergoing chemotherapy) }\end{array}$ \\
\hline Defective oxidative burst & $\begin{array}{l}\text { Chronic granulomatous disease, myeloperoxidase deficiency and G6PD } \\
\text { deficiency }\end{array}$ \\
\hline Defective chemotaxis & $\begin{array}{l}\text { Leukocyte adhesion deficiency type I, Wiskott-Aldrich syndrome and RAC2 } \\
\text { deficiency }\end{array}$ \\
\hline Granule disorders & Neutrophil-specific granule deficiency and Chediak-Higashi syndrome \\
\hline $\begin{array}{l}\text { Combined defects in oxidative } \\
\text { burst, chemotaxis and phagocytosis }\end{array}$ & $\begin{array}{l}\text { Diabetes mellitus and renal insufficiency (in particular, patients on } \\
\text { haemodialysis) }\end{array}$ \\
\hline \multicolumn{2}{|l|}{ Signalling } \\
\hline Defects in IL-1R or TLR signalling & MYD88 deficiency and IRAK4 deficiency \\
\hline \multicolumn{2}{|l|}{ T cells } \\
\hline \multirow[t]{3}{*}{ Decreased $T_{H} 17$ cell numbers } & $\begin{array}{l}\text { Hyper-lgE syndrome (caused by STAT3 and DOCK } 8 \text { mutations that render } \\
\text { patients deficient of } \mathrm{T}_{\mathrm{H}} 17 \text { cells) }\end{array}$ \\
\hline & $\begin{array}{l}\text { Atopic dermatitis (caused by skin barrier defects, including filaggrin mutations, } \\
\text { that lead to decreased levels of antimicrobial peptides, increased } \mathrm{T}_{\mathrm{H}} 2 \text { cell } \\
\text { responses and decreased } \mathrm{T}_{\mathrm{H}} 17 \text { cell responses) }\end{array}$ \\
\hline & $\begin{array}{l}\text { HIV/AIDS (which results in decreased numbers of } C D 4^{+} \mathrm{T} \text { cells, including } \\
\mathrm{T}_{H} 17 \text { cells) }\end{array}$ \\
\hline $\begin{array}{l}\text { IL-17F and IL-17RA deficiency } \\
\text { (or patients with autoantibodies } \\
\text { specific for IL-17A, IL-17F and IL-22) }\end{array}$ & $\begin{array}{l}\text { Chronic mucocutaneous candidiasis (in which patients have increased } \\
\text { susceptibility mainly to mucocutaneous Candida infections, but also to S. aureus } \\
\text { skin infections) }\end{array}$ \\
\hline
\end{tabular}

DOCK8, dedicator of cytokinesis 8; G6PD, glucose-6-phosphate dehydrogenase; IL, interleukin; IL-1R, IL-1 receptor; IL-17RA, IL-17 receptor A; IRAK4, IL-1R-associated kinase 4; MYD88, myeloid differentiation primary response protein 88; STAT3, signal transducer and activator of transcription $3 ; \mathrm{T}_{\mathrm{H}}$, Thelper; TLR, Toll-like receptor.
Pathogen-associated molecular patterns (PAMPs). Small conserved molecular motifs found in components of microorganisms that are not present in mammalian cells. Examples include bacterial lipopeptides, lipoteichoic acid and peptidoglycan, which activate pattern recognition receptors (such as Toll-like receptors) on host cells

\section{Oxidative burst}

The rapid production of reactive oxygen species in phagocytic vacuoles of neutrophils and other myeloid cells. There are two main enzymes involved in the production of reactive oxygen species: NADPH oxidase (which generates superoxide) and myeloperoxidase (which combines hydrogen peroxide with chloride ions to produce hypochlorous acid). Although these reactive oxygen species can kill bacteria directly, they also produce a charge across the phagosomal membrane that facilitates the enzymatic digestion and killing of microorganisms. (such as granulocyte colony-stimulating factor (G-CSF) and granulocyte-macrophage colony-stimulating factor (GM-CSF)), which stimulate neutrophil production $^{44,45}$. In addition, a subset of $\mathrm{T}$ cells that produces IL-22 but not IL-17 also contributes to cutaneous immune responses ${ }^{46-48}$. Differentiation of $\mathrm{T}_{\mathrm{H}} 17$ cells occurs in response to the signal transducer and activator of transcription 3 (STAT3)-activating cytokines IL-6, IL-21 and IL-23, in the presence of transforming growth factor- $\beta$ (TGF $\beta$ ) and IL- $1 \beta^{44,45}$. Both IL-17 and IL-22 have been shown to induce keratinocyte production of antimicrobial peptides (such as the human cathelicidin LL-37, human $\beta$-defensin 2 and psoriasin (also known as $\mathrm{S} 100 \mathrm{~A} 7))^{49,50}$, and IL-21 and IL-22 promote hyperproliferation of the epidermis, which is important in wound healing ${ }^{48,51}$. Although $\mathrm{T}_{\mathrm{H}} 17$ cells are involved in many of these different cutaneous immune responses, this Review focuses on $\mathrm{T}_{\mathrm{H}} 17$ cell responses that promote host defence against $S$. aureus skin infections.

\section{Diseases associated with S. aureus infections}

Defects in the number or function of neutrophils can result in an increased susceptibility to $S$. aureus infections in various tissues and organs ${ }^{52-55}$. However, certain genetic and acquired immunodeficiency conditions that primarily result in an increased susceptibility to $S$. aureus infections at epithelial sites have been found to result from signalling defects in IL-1R or TLR pathways ${ }^{17-20}$ or from $\mathrm{T}$ cell defects ${ }^{21-24}$. These diseases have provided important insights into the immune response against $S$. aureus infections in the skin (TABLE 1).

Defects in neutrophil function or recruitment. Defects in neutrophil number or function (such as oxidative burst, chemotaxis and granule disorders) result in an increased susceptibility to $S$. aureus infections in a wide variety of tissues and organs ${ }^{52-55}$ (TABLE 1). Decreased neutrophil numbers are seen in genetic immunodeficiency conditions such as severe congenital neutropenia, in which neutrophil development is prematurely arrested, and in acquired neutropenia, which is seen in cancer patients undergoing chemotherapy ${ }^{52,53,55}$. Diseases involving an impaired oxidative burst (that is, defects in the ability to generate reactive oxygen species) include chronic granulomatous disease (which is characterized by defects in NADPH oxidase, the enzyme responsible for generating $\mathrm{O}_{2}^{-}$) and myeloperoxidase deficiency (myeloperoxidase combines $\mathrm{H}_{2} \mathrm{O}_{2}$ with $\mathrm{Cl}^{-}$to generate $\left.\mathrm{HOCl}\right)^{53-55}$. Reactive oxygen species can mediate direct killing of microorganisms, but they also produce a charge in the membrane of the phagocytic vacuole that promotes enzymatic digestion and killing of pathogens ${ }^{42}$.

Conditions characterized by defective neutrophil chemotaxis include leukocyte adhesion deficiency type I, which is caused by a deficiency in the integrin subunit CD18, and Wiskott-Aldrich syndrome and RAC2 deficiency, which involve dysfunctional actin polymerization and cytoskeletal rearrangements, respectively ${ }^{53-55}$. 
Diseases resulting from neutrophil granule impairments include neutrophil-specific granule deficiency, which is characterized by a lack of secondary granule proteins such as antimicrobial peptides ( $\alpha$-defensins, for example), and Chediak-Higashi syndrome, in which the neutrophils have giant azurophil granules with impaired antimicrobial activity ${ }^{53,55}$. Finally, patients with diabetes mellitus and renal insufficiency have a combination of defects in neutrophil oxidative burst, chemotaxis and phagocytosis ${ }^{56,57}$.

Defects in innate immune signalling molecules. In contrast to neutrophil defects, which result in systemic susceptibility to $S$. aureus, paediatric patients who are deficient in the signalling molecules MYD88 or IRAK4 have a predisposition to 'pyogenic' infections (which are characterized by neutrophil abscess formation) at epithelial sites ${ }^{18,19}$ (TABLE 1). IL-1R and TLR family members signal through MYD88, which subsequently interacts with IRAK4, to activate TNFR-associated factor 6 (TRAF6). This induces the nuclear factor- $\kappa B$ (NF- $\mathrm{kB}$ ) and mitogen-activated protein kinase (MAPK) signalling pathways, leading to the transcription of proinflammatory genes (such as those encoding TNF, IL-6 and CXCL8) ${ }^{18,19}$ (FIG. 2). The phenotypes of MYD88- and IRAK4-deficient patients were shown to be identical: 80\% developed Streptococcus pneumoniae pneumonia, one-third suffered from severe $S$. aureus skin infections, and other infections were surprisingly rare ${ }^{17-20}$. About half of these patients die before they are 8 years old (mostly from meningitis and sepsis), and the frequency of S. pneumoniae and S. aureus infections decreases with age, with no infections seen in patients who are more than 14 years old ${ }^{17-20}$. As these infections depend on age, it is probable that both innate and adaptive immune responses contribute to host defence against $S$. aureus. In early childhood, when innate immunity predominates, signalling through IL-1R and TLR family members

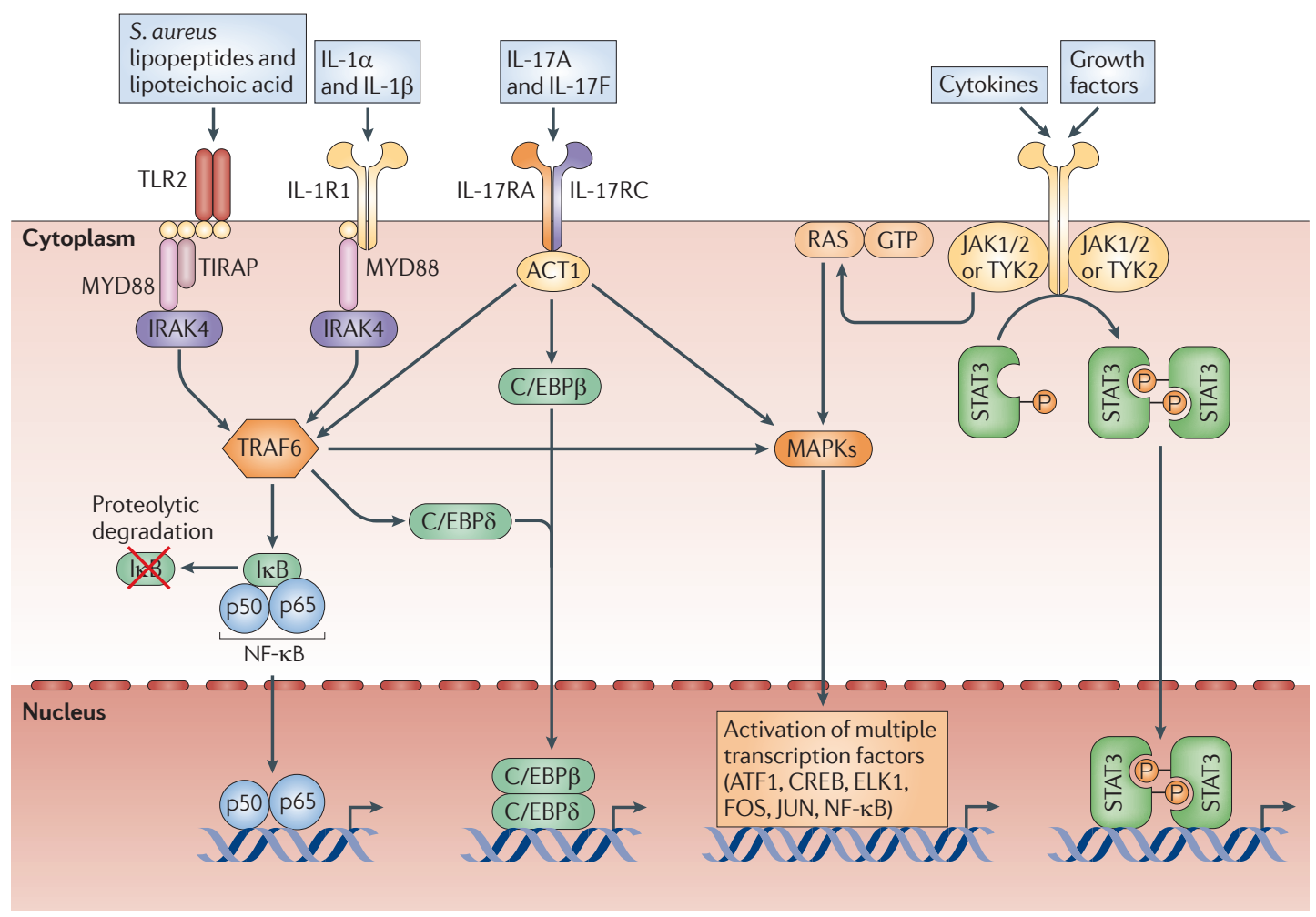

Figure 2 | Major host cell signalling pathways involved in the immune response against S. aureus cutaneous infections. Toll-like receptor 2 (TLR2), which is activated by Staphylococcus aureus lipopeptides and lipoteichoic acid, and interleukin-1 receptor 1 (IL-1R1), which is activated by IL-1 $\alpha$ and IL-1 $\beta$, both signal through the adaptor molecule MYD88 (myeloid differentiation primary response protein 88). MYD88 triggers the activation of IRAK4 (IL-1R-associated kinase 4) and TRAF6 (TNFR-associated factor 6 ), leading to the activation of NF- $\kappa \mathrm{B}$ (nuclear factor- $\kappa \mathrm{B}), \mathrm{C} / \mathrm{EBP} \beta / \delta$ (CCAAT/ enhancer-binding protein- $\beta / \delta$ ) and MAPKs (mitogen-activated protein kinases, such as JNK (JUN N-terminal kinase), p38 and ERK1 (extracellular signal-regulated kinase 1) or ERK2). TLR2 also uses TIRAP (TIR domain-containing adaptor protein) to initiate signalling. The IL-17RA-IL-17RC complex, which is expressed mainly on epithelial cells, is activated by IL-17A and IL-17F homodimers or heterodimers and uses ACT1 (NF- $\mathrm{kB}$ activator 1 ) to activate similar pathways to MYD88. STAT3 (signal transducer and activator of transcription 3) is a transcription factor that is activated downstream of various cytokine (IL-6, IL-10, IL-11, IL-19, IL-21, IL-22, IL-23, IL-24, IL-25, IL-26, IL-27 and IL-35) receptors and growth factor receptors (EGFR, FGFR, IGFR, HER2, HGFR, PDGFR, VEGFR and GCSFR). Each of these signalling pathways leads to the activation of transcription factors that alone or in combination promote the transcription of pro-inflammatory mediators involved in the immune response against S. aureus. ATF1, activating transcription factor 1; CREB, cAMP-responsive

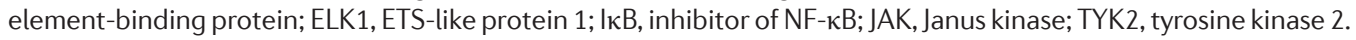


would be mainly responsible for inducing the protective neutrophilic response against $S$. aureus skin infections. This is consistent with the lack of an increase in blood neutrophil counts during infection in younger patients with MYD88 or IRAK4 deficiency ${ }^{58}$. However, with age, the adaptive immune responses that develop in the absence of signals through TLRs and IL-1R can protect against $S$. aureus cutaneous infections ${ }^{58}$.

Defects in T cells. There are several human diseases in which an association between $\mathrm{T}$ cell dysfunction and susceptibility to $S$. aureus cutaneous infections has been shown (TABLE 1). Patients with autosomal dominant and recessive forms of hyper-IgE syndrome, who suffer from recurrent and severe $S$. aureus cutaneous infections, were found to have STAT3 (REFS 59,60) and dedicator of cytokinesis 8 (DOCK 8$)^{61,62}$ mutations, respectively, that render them deficient in $\mathrm{T}_{\mathrm{H}} 17$ cells ${ }^{21-24}$. Indeed, signalling mediated by multiple $\mathrm{T}_{\mathrm{H}} 17$ cell-associated cytokines - for example, IL-6, IL-21 and IL-23 - depends on STAT3 (REF. 63) (FIG. 2). STAT3-dependent signalling is also used by numerous growth factors that have an important role in wound healing, such as epidermal growth factor (EGF) and fibroblast growth factor (FGF) ${ }^{63}$.

In addition, patients with atopic dermatitis have increased colonization and superinfection with S. aureus. This has been attributed to the skin barrier dysfunction, as well as the enhanced $\mathrm{T}_{\mathrm{H}} 2$ cell responses, decreased production of antimicrobial peptides and decreased $\mathrm{T}_{\mathrm{H}} 17$ cell responses, in this disease $\mathrm{e}^{64-66}$. Patients with HIV infection with low $\mathrm{CD}^{+} \mathrm{T}$ cell counts are also predisposed to $S$. aureus cutaneous infections ${ }^{67}$, and this might be the result of a loss of $\mathrm{T}_{\mathrm{H}} 17$ cells $^{68}$. Finally, humans with chronic mucocutaneous candidiasis, who suffer from candidal infections and to a lesser degree S. aureus skin infections, were found to have autoantibodies specific for the $\mathrm{T}_{\mathrm{H}} 17$ cell cytokines IL-17A, IL-17F and IL-22, as well as mutations in the genes encoding IL-17F and IL-17 receptor A (IL-17RA) ${ }^{69-71}$. IL-17A and IL-17F signal through the IL-17RA-IL-17RC complex to activate NF- $\kappa B$ activator 1 (ACT1; also known as CIKS and TRAF3IP2), which triggers TRAF6-, CCAAT/enhancerbinding protein- $\beta / \delta(\mathrm{C} / \mathrm{EBP} \beta / \delta)$ - and MAPK-induced transcription of pro-inflammatory genes that are important for neutrophil recruitment and the production of antimicrobial peptides at epithelial sites ${ }^{72}$ (FIG. 2). In summary, although patients with neutrophil dysfunction are predisposed to systemic $S$. aureus infections, patients with defective IL-1R and/or TLR signalling or defective $\mathrm{T}_{\mathrm{H}} 17$ cell responses have an increased susceptibility to S. aureus infection in the skin.

Chronic mucocutaneous candidiasis

A heterogenous group of disorders that share in

common an immune defect that predisposes individuals to recurrent or persistent infections of the skin, mucous membranes and nails with candidal species such as Candida albicans. through TRIF rather than MYD88), as well as the IL-1R family members IL-1R, IL-18R and IL-33R ${ }^{37,58}$. The specific defects in receptor signalling that are responsible for the increased susceptibility to infection seen in MYD88- or IRAK4-deficient patients have not been identified ${ }^{58}$. Although there might be differential contributions by each of these receptors ${ }^{58}$, two receptors that have been studied the most in the context of $S$. aureus cutaneous infections are TLR2 and IL-1R.

TLR2 versus IL-1R. TLR2 heterodimerizes with either TLR1 or TLR6 to recognize bacterial triacyl or diacyl lipopeptides, respectively, and it has been shown to recognize $S$. aureus lipopeptides and lipoteichoic acid ${ }^{37}$. By contrast, IL-1R is activated by IL- $1 \alpha$ and IL- $1 \beta$, which are produced by host cells ${ }^{15}$. Using a mouse model of $S$. aureus skin infection, our laboratory found that MYD88-deficient mice had an increased susceptibility to infection compared with wild-type mice owing to severe impairments in bacterial clearance, neutrophil recruitment and the production of neutrophil-attracting chemokines ${ }^{25}$. To determine the predominant MYD88-dependent receptor that contributed to this phenotype, the immune responses in IL-1R- and TLR2-deficient mice were compared ${ }^{25}$. IL-1R-deficient mice, but not TLR2-deficient mice, had similar defects in bacterial clearance and neutrophil recruitment to MYD88-deficient mice ${ }^{25}$. These findings indicate that IL-1R-MYD88 signalling is a more important determinant than TLR2-MYD88 signalling for neutrophil recruitment and host defence against $S$. aureus cutaneous infections. Although IL-1R-deficient patients have not yet been identified, a similar role for IL-1R signalling in host defence against $S$. aureus skin infections has been suggested in humans. In patients with rheumatoid arthritis who are treated with recombinant IL-1 receptor antagonist (IL-1RA; which inhibits IL-1R activity), it has been reported that the incidence of $S$. aureus skin infections increases with higher doses of the drug ${ }^{73}$.

$I L-1 \alpha$ versus $I L-1 \beta$. Although IL- $1 \alpha$ and IL- $1 \beta$ both signal through IL-1R, their cellular source and posttranslational processing are different ${ }^{15}$. In the skin, pre-made stores of biologically active IL-1 $\alpha$ are present in keratinocytes and are released following nonspecific injury or infection $^{15}$ (FIG. 3a). By contrast, IL-1 $\beta$ is an inducible cytokine that is produced by many cell types, including keratinocytes and immune cells such as macrophages and dendritic cells ${ }^{15}$. Our laboratory found that, following infection of a superficial skin wound with $S$. aureus, mice deficient in either IL-1a or IL- $1 \beta$ had only slightly impaired bacterial clearance compared with control mice, in contrast to the severe infections seen in IL-1R-deficient mice ${ }^{74}$. However, when the same IL-1 $\alpha$ - or IL- $1 \beta$-deficient mice were inoculated intradermally with $S$. aureus, bacterial clearance in IL-1 $\beta$-deficient mice was impaired to a similar extent as that in IL-1R-deficient mice, whereas IL-1 $\alpha$-deficient mice had wild-type levels of bacterial 
a

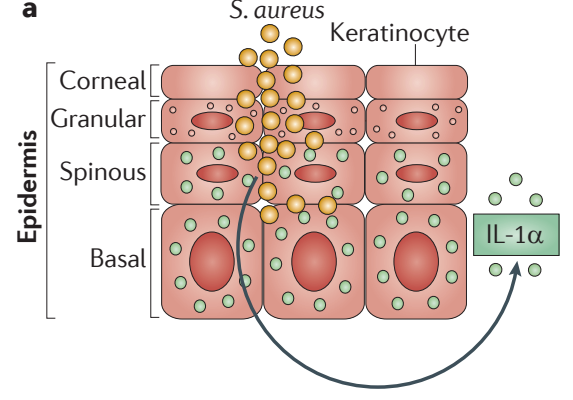

b

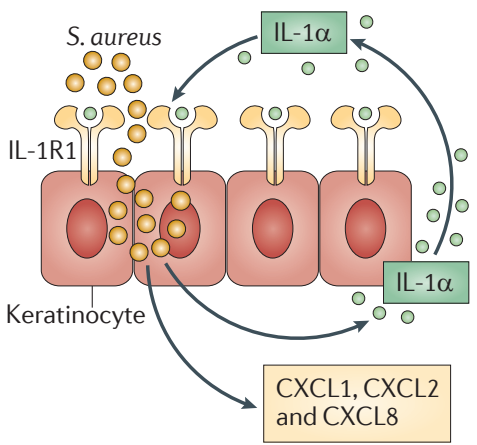

C

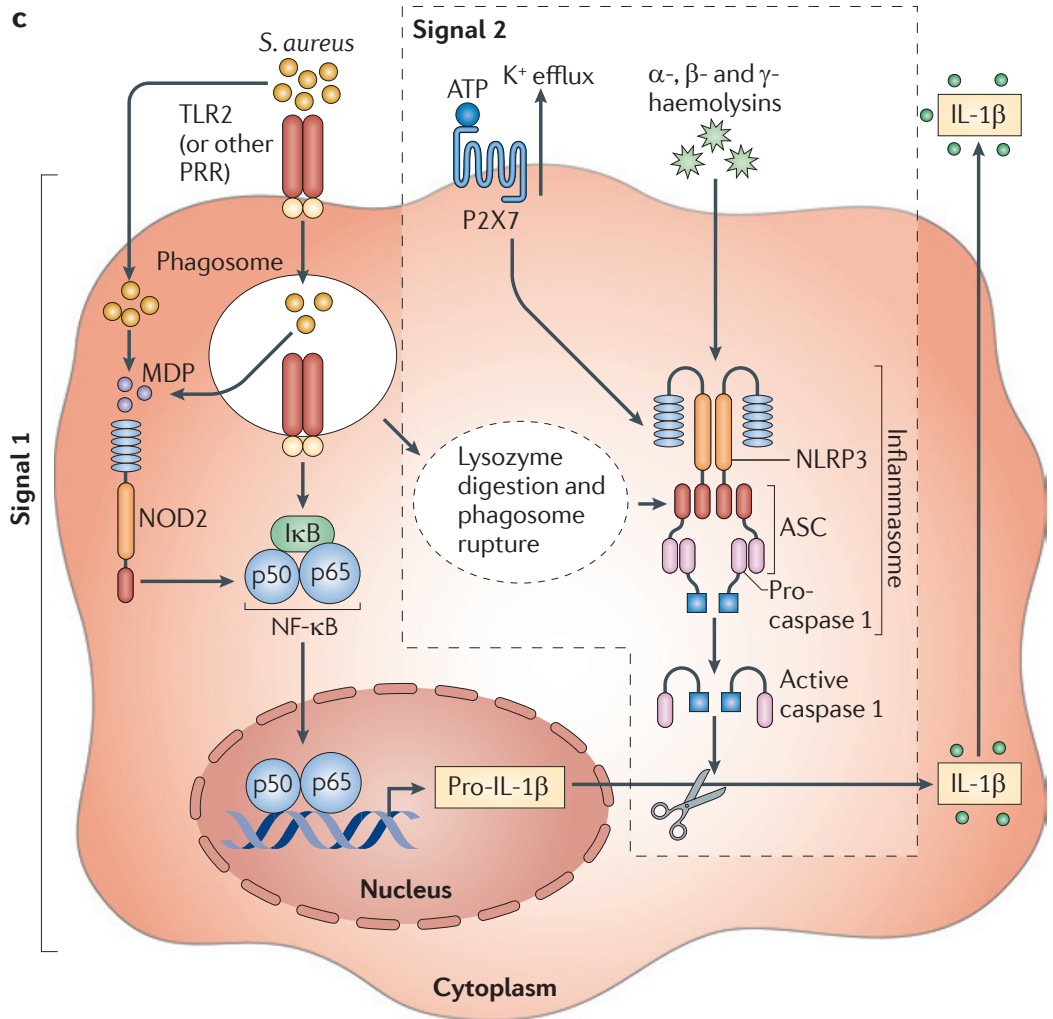

Figure $3 \mid$ Mechanisms of IL-1 $\alpha$ and IL-1 $\beta$ production during S. aureus

cutaneous infections. a | Epidermal keratinocytes constitutively contain stores of pre-made interleukin-1a (IL-1 $\alpha$ ) during resting conditions, which is rapidly released in response to nonspecific injury, inflammation or infection. $\mathbf{b} \mid$ In keratinocytes, Staphylococcus aureus induces an IL-1a-mediated autocrine signalling loop through IL-1 receptor 1 (IL-1R1), which leads to the rapid and continuous production of neutrophil-attracting chemokines, such as CXC-chemokine ligand 1 (CXCL1), CXCL2 and CXCL8. c| The production of IL-1 $\beta$ during S. aureus cutaneous infections requires two signals. Signal 1 involves the transcription of pro-IL-1 $\beta$, which is induced by the activation of pattern recognition receptors (PRRs), such as TLR2 (Toll-like receptor 2) and NOD2 (nucleotide-binding oligomerization domain-containing protein 2). Signal 2 involves NLRP3 (NOD-, LRR- and pyrin domain-containing 3 ) inflammasome activation, which results in caspase 1 -mediated cleavage of pro-IL-1 $\beta$ into active IL-1 $\beta$. NLRP3 inflammasome activation during $S$. aureus infections has been shown to be triggered by lysozyme-mediated digestion of S. aureus (which releases peptidoglycan, induces the production of reactive oxygen intermediates and promotes phagosome rupture), by the activation of the purinergic receptor P $2 X 7$ by ATP (which is released by stressed or damaged cells during an infection) and by pore-forming toxins of $S$. aureus, such as the $\alpha-, \beta$ - and $\gamma$-haemolysins. I $\kappa B$, inhibitor of NF- $\kappa B$; MDP, muramyl dipeptide; NF- $\kappa B$, nuclear factor- $\kappa B$. clearance $^{74}$. So, both IL- $1 \alpha$ and IL- $1 \beta$ contribute to host defence during a superficial $S$. aureus skin infection, whereas IL- $1 \beta$ has a predominant role during a deeper $S$. aureus skin infection ${ }^{74}$. In human keratinocytes, $S$. aureus was found to induce the release of substantially more IL- $1 \alpha$ than IL- $1 \beta$ and initiated an IL- $1 \alpha-$ IL-1R signalling loop in human keratinocytes that led to the continuous production of neutrophil-attracting chemokines, such as CXCL1, CXCL2 and CXCL8 (REF. 75) (FIGS 3b,4). Taken together, these data indicate that IL-1 $\alpha$ has a more important role during S. aureus colonization and superficial infections, such as impetigo and infected abrasions, whereas IL- $1 \beta$ is more crucial during invasive skin infections, such as cellulitis.

Inflammasome activation. The production of IL-1 $\beta$ is tightly controlled and typically requires two separate signals (FIG. 3c). The first signal involves activation of PRRs, such as TLR2, leading to NF- $\kappa B$-mediated transcription of the gene encoding pro-IL- $1 \beta^{76}$. During $S$. aureus cutaneous infection, TLR2-deficient mice had impaired production of IL- $1 \beta$, but IL- $1 \beta$ was still produced to some extent, which indicates that other PRRs are also involved in IL- $1 \beta$ production ${ }^{25}$. For example, mice deficient in NOD2 (which recognizes muramyl dipeptide, a breakdown product of $S$. aureus peptidoglycan) also had impaired production of proIL- $1 \beta$ during cutaneous infection with $S$. aureus ${ }^{77}$. In this model, IL- $1 \beta$ induced the production of IL- 6 , which enhanced neutrophil antimicrobial mechanisms against S. aureus $^{77}$. So, both TLR2 and NOD2 induce pro-IL-1 $\beta$ production and promote host defence against $S$. aureus cutaneous infections.

The second signal required for the production of IL- $1 \beta$ initiates the proteolytic processing of pro-IL- $1 \beta$ by caspase 1 to generate active and secreted IL-1 $\beta^{76}$ (FIG. 3c). This processing depends on the formation of an intracellular complex of proteins known as the inflammasome ${ }^{76}$. There are several types of inflammasome, each containing a different complex of proteins. These inflammasome types include the NLRP1 (NOD-, LRR- and pyrin domain-containing 1), the NLRP3, the NLRC4 (NOD-, LRR- and CARD-containing 4; also known as IPAF) and the AIM2 (absent in melanoma 2) inflammasomes ${ }^{76}$. In $S$. aureus-stimulated mouse macrophage cultures, inflammasome activation was found to depend on the adaptor protein ASC, which is a component of most inflammasomes ${ }^{78}$. Similarly, during $S$. aureus cutaneous infection, ASC-deficient mice had the same impairments in host defence and bacterial clearance as IL- $1 \beta$-deficient mice ${ }^{26}$. Thus, ASC-dependent inflammasome activation is required to generate active IL-1 $\beta$ during $S$. aureus cutaneous infections. Several mechanisms have been shown to promote NLRP3 inflammasome activation in the context of an $S$. aureus infection, namely: triggering of the purinergic receptor P2X7 by increased levels of ATP (which is released by stressed or damaged cells during an infection $)^{79}$; pore formation mediated by the $\alpha-, \beta$ and $\gamma$-haemolysins of $S$. aureus ${ }^{27,80}$; and the digestion of peptidoglycan mediated by lysozyme ${ }^{81}$. 


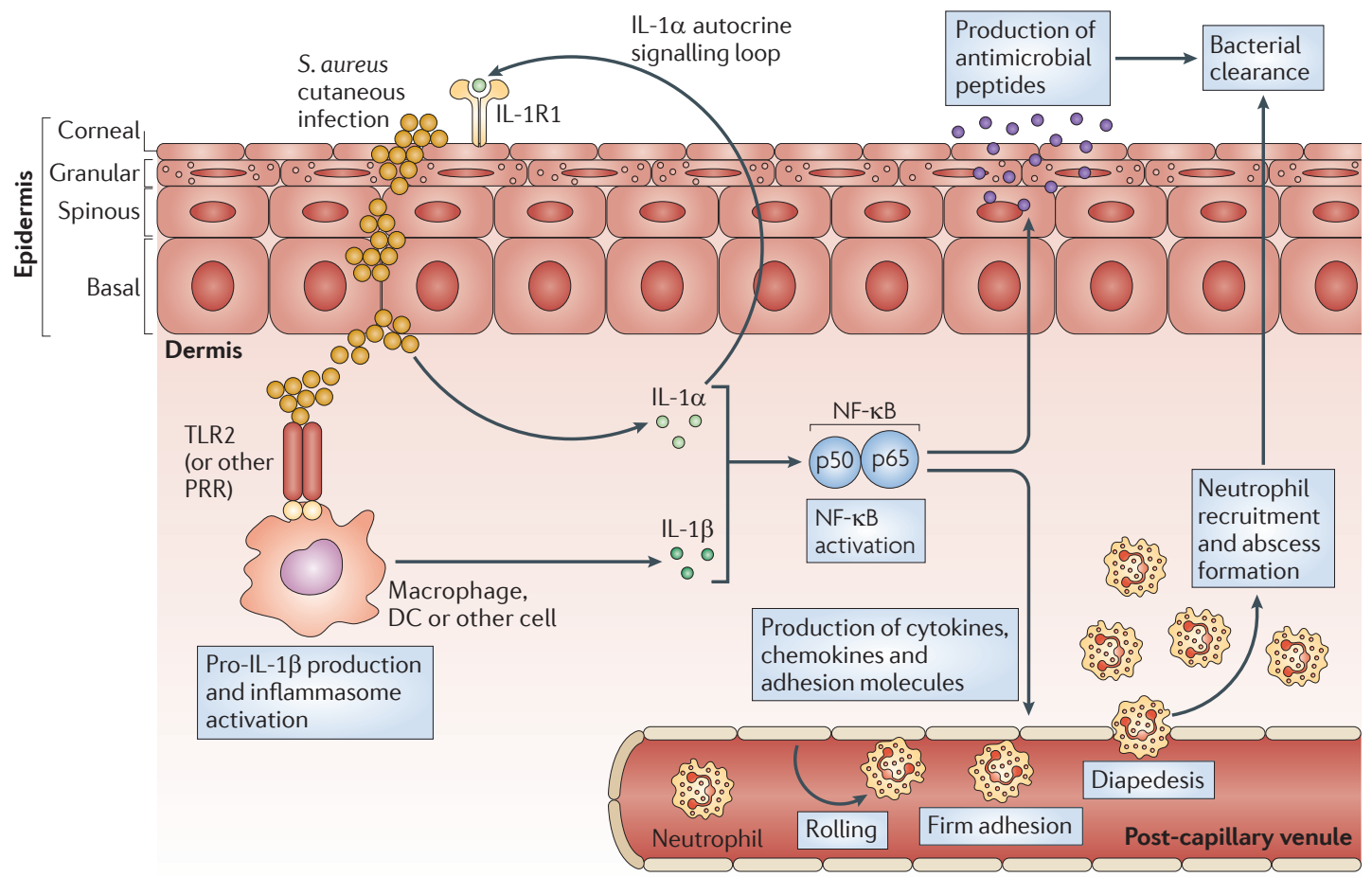

Figure 4 | The IL-1-mediated cutaneous immune response against S. aureus. Interleukin-1 $\alpha$ (IL-1 $\alpha$ ), which is produced and released by keratinocytes, and IL-1 $\beta$, which is produced by resident and recruited cells (such as macrophages and dendritic cells (DCs)), trigger the activation of nuclear factor- $\mathrm{KB}(\mathrm{NF}-\kappa \mathrm{B})$ (and of other important pro-inflammatory signalling molecules, such as $C / E B P \beta / \delta$ (CCAAT/enhancer binding protein- $\beta / \delta$ ) and MAPKs (mitogen-activated protein kinases)). These signalling pathways lead to the production of antimicrobial peptides (such as $\beta$-defensins 2 and 3 , cathelicidins and RNase 7) that have bacteriostatic and bactericidal activity against Staphylococcus aureus. IL-1-mediated responses also result in the production of pro-inflammatory cytokines, chemokines and adhesion molecules that promote the recruitment of neutrophils from the circulation to the site of S. aureus infection in the skin. Recruited neutrophils form an abscess to help control and limit the spread of the infection, and this is ultimately required for bacterial clearance. TLR2, Toll-like receptor 2.

Inflammasome An intracellular complex of proteins that promotes the activation of caspase 1 , which cleaves pro-interleukin-1 $\beta$ (pro-IL-1 $\beta$ ) into the active and secreted form of IL- $1 \beta$.

$\gamma \delta$ T cells

T cells that express the $\gamma$ - and $\delta$-chains of the $T$ cell receptor. $\gamma \delta \mathrm{T}$ cells are found mainly at epithelial sites, have invariant $\mathrm{T}$ cell receptors and can rapidly participate in immune responses as they do not require priming in lymphoid tissues.
Proposed role of IL-1 in S. aureus skin infections. In summary, these studies have elucidated an immune defence pathway beginning with $S$. aureus-induced activation of an IL-1 $\alpha$ autocrine signalling loop involving keratinocytes and of PRR- and inflammasome-mediated production of IL- $1 \beta$ by keratinocytes and immune cells. These two pathways together trigger IL-1R-MYD88 signalling (FIG. 4). IL-1R-MYD88 signalling activates NF- $\kappa B$ (FIG. 2), together with other signalling pathways, and this results in the production of pro-inflammatory cytokines, chemokines and adhesion molecules that mediate neutrophil recruitment (and antimicrobial peptide production) to promote bacterial clearance.

\section{Role of IL-17 in cutaneous S. aureus infection}

Although humans with hyper-IgE syndrome, atopic dermatitis, HIV/AIDS and mucocutaneous candidiasis are known to have increased susceptibility to $S$. aureus skin infections, the mechanisms were thought to be unrelated until recently. Emerging data indicate that these conditions have in common a deficiency of $\mathrm{T}_{\mathrm{H}} 17$ cells ${ }^{21-24,64,68-71}$ (TABLE 1), which indicates that $T_{H} 17$ cells have a role in host defence against $S$. aureus at cutaneous sites of infection.

One study sought to explain why patients with autosomal dominant hyper-IgE syndrome are predisposed to $S$. aureus infections in the skin and lungs but not at other sites ${ }^{82}$. They found that human keratinocytes and bronchial epithelial cells have a much stronger dependence on IL-17 and IL-22 (together with IL-1 $\beta$ ) for the induction of neutrophil-attracting chemokines and antimicrobial peptides than do other cell types (such as fibroblasts and endothelial cells) ${ }^{82}$. Thus, this preferential activity of IL-17 and IL-22 on keratinocytes and bronchial epithelial cells indicates that $\mathrm{T}_{\mathrm{H}} 17$ cell responses are particularly relevant for promoting host defence against $S$. aureus in the skin and lungs.

IL-17 has also been implicated in host defence against S. aureus cutaneous infections in vivo. Mice deficient in both IL-17A and IL-17F develop spontaneous S. aureus skin infections but do not have an increased susceptibility to intravenous $S$. aureus challenge ${ }^{28}$. Our laboratory found that in a mouse model of cutaneous $S$. aureus infection, mice deficient in $\gamma \delta$ T cells developed larger skin lesions and had increased bacterial counts, decreased production of neutrophil-attracting chemokines and impaired neutrophil recruitment compared with wildtype mice ${ }^{29}$. The defective neutrophilic response in $\gamma \delta$ T cell-deficient mice was the result of a lack of early production of IL-17A and IL-17F (within 8 hours of the infection), a process that is mediated by resident epidermal $\gamma \delta$ T cells in wild-type mice and which requires 


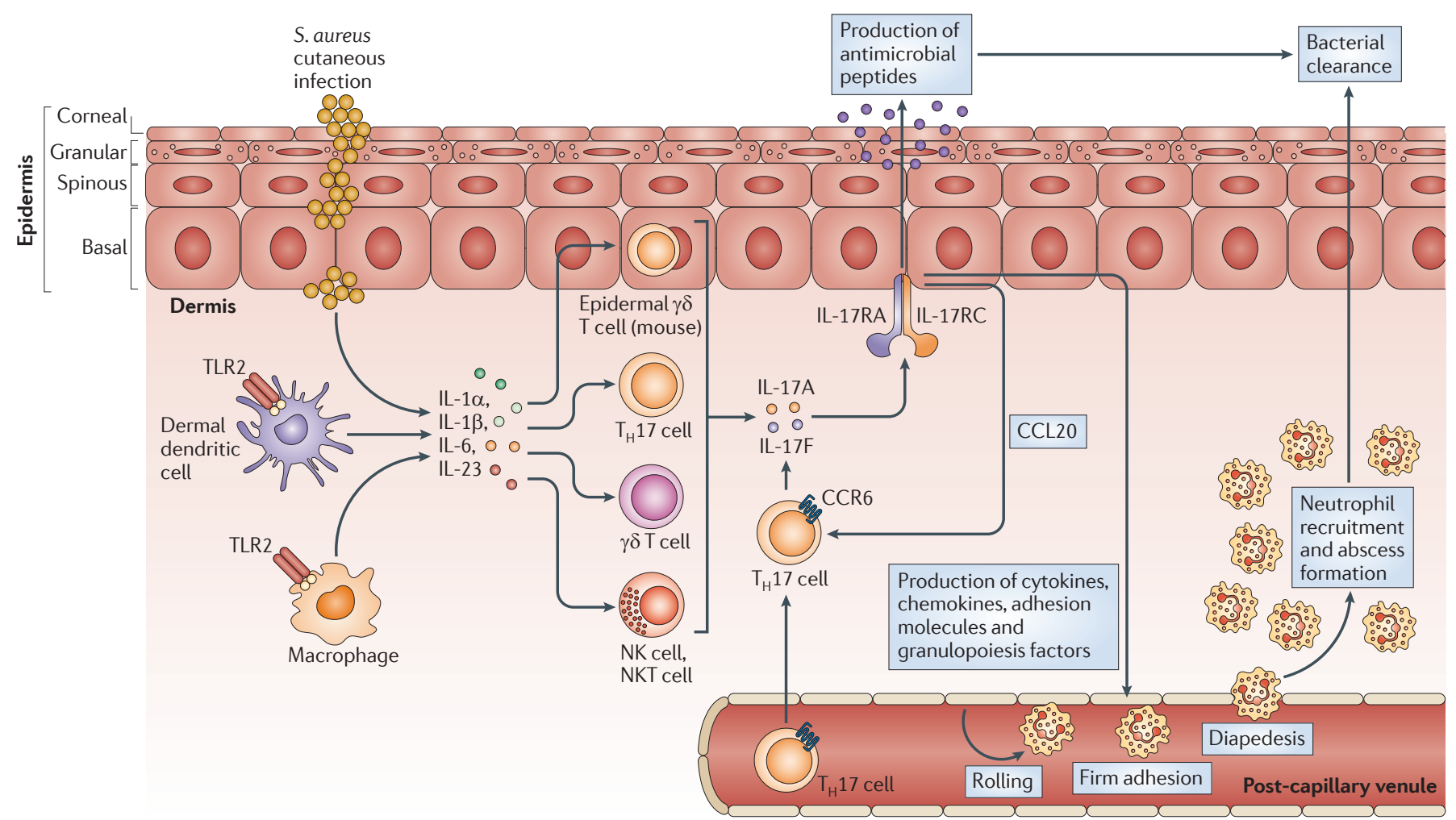

Figure 5 | The IL-17-mediated cutaneous immune response against S. aureus. Interleukin-17A (IL-17A) and IL-17F are produced by various cells in the skin, including $\gamma \delta$ T cells in the epidermis (in mice) and Thelper $17\left(\mathrm{~T}_{H} 17\right)$ cells, $\gamma \delta$ T cells, natural killer (NK) cells and NKT cells in the dermis. The production of IL-17A and IL-17F depends on the activation of Toll-like receptor 2 (TLR2) and the production of IL-1 $\alpha$, IL-1 $\beta$, IL-6 and IL-23, which can be produced by other resident and recruited cells in the skin, such as macrophages and dendritic cells. IL-17A and IL-17F activate IL-17 receptors (IL-17RA-IL-17RC) on epidermal keratinocytes, resulting in the production of antimicrobial peptides (such as $\beta$-defensins 2 and 3, and cathelicidins) that have bacteriostatic and bactericidal activity against Staphylococcus aureus, as well as in the production of pro-inflammatory cytokines, chemokines and adhesion molecules that promote the recruitment of neutrophils from the circulation to the site of S. aureus infection in the skin. IL-17-mediated signalling also leads to the production of GM-CSF (granulocyte-macrophage colony-stimulating factor) and G-CSF, which promote neutrophil granulopoiesis in the bone marrow and perhaps locally in the skin. Neutrophils form an abscess to help control and limit the spread of the infection in the skin, and this is ultimately required for bacterial clearance. IL-17-mediated signalling also leads to the production of CC-chemokine ligand 20 (CCL20), which triggers a positive feedback loop by promoting additional recruitment from the circulation of $\mathrm{T}_{\mathrm{H}} 17$ cells that preferentially express $C$ C-chemokine receptor 6 (CCR6).

IL-1 $\beta$, IL-23 and TLR2 (REF. 29). The key role for IL-17 in host defence against $S$. aureus cutaneous infection in this mouse model was confirmed by the impaired bacterial clearance observed in IL-17R-deficient mice and in wildtype mice treated with an IL-17A-specific neutralizing antibody ${ }^{29}$. However, as $\gamma \delta$ T cells have not been identified in the human epidermis, it is likely that $\mathrm{T}_{\mathrm{H}} 17$ cells, $\gamma \delta$ T cells and NK cells that are present in the dermis of human skin ${ }^{16}$ are functional equivalents for the production of IL-17 and other $\mathrm{T}_{\mathrm{H}} 17$ cell-related cytokines to protect against $S$. aureus infection in human skin. In fact, there are approximately $2 \times 10^{10}$ resident $\mathrm{T}$ cells in human skin, which is twice the number of $\mathrm{T}$ cells in the blood ${ }^{83}$. Of note, two studies recently identified a $\gamma \delta \mathrm{T}$ cell population in the dermis of mouse skin that is capable of rapidly producing IL-17 (REFS 84,85). It will be interesting to determine whether these cells contribute to host defence against $S$. aureus skin infections and whether a similar population exists in human skin.
Taken together, these studies demonstrate the important role of IL-17-mediated responses in neutrophil recruitment and host defence against $S$. aureus cutaneous infections, providing an explanation for the increased susceptibility to $S$. aureus skin infections in patients with hyper-IgE syndrome, atopic dermatitis, HIV/AIDS and mucocutaneous candidiasis.

Proposed role of IL-17 in S. aureus skin infections. These studies indicate a model of immune defence beginning with $S$. aureus-induced TLR2 activation and the production of IL- $1 \alpha$, IL- $1 \beta$, IL- 6 and IL-23, which stimulate IL-17-producing $\mathrm{T}$ cells in the skin (such as $\gamma \delta \mathrm{T}$ cells in the epidermis and $\mathrm{T}_{\mathrm{H}} 17$ cells, $\gamma \delta \mathrm{T}$ cells, NK cells and NKT cells in the dermis) (FIG. 5). IL-17A and IL-17F then stimulate keratinocytes to produce pro-inflammatory cytokines, chemokines and adhesion molecules that mediate neutrophil recruitment to the site of infection in the skin (as well as to produce 
antimicrobial peptides) to promote bacterial clearance. In addition, IL-17A and IL-17F induce keratinocyte production of CC-chemokine ligand 20 (CCL20), which binds to CC-chemokine receptor 6 (CCR6) and provides a positive feedback loop that leads to the recruitment of additional $\mathrm{T}_{\mathrm{H}} 17$ cells that preferentially express CCR6 (REFS 86,87).

\section{Potential immunotherapy against $S$. aureus}

The recent discovery of the important roles of IL-1 and IL-17 in neutrophil recruitment and antimicrobial peptide production indicates that strategies aimed at enhancing these responses might be of prophylactic and therapeutic use against $S$. aureus cutaneous infections in humans.

Current strategies. Most immune-based strategies against $S$. aureus infections have been antibody based to enhance opsonization of the bacteria, which promotes phagocytosis and clearance ${ }^{88,89}$. Such strategies have included vaccination against $S$. aureus capsular polysaccharides 5 and 8 (StaphVax; Nabi Pharmaceuticals) and an immunoglobulin preparation against $S$. aureus clumping factor A (Veronate; Inhibitex, Inc.). However, these approaches have ultimately had limited efficacy in clinical trials, despite promising results in preclinical animal models ${ }^{88,89}$. Interestingly, a recent study found that antibodies specific for $S$. aureus capsular polysaccharides and the surface polysaccharide poly- $N$-acetylglucosamine, which independently demonstrated protection in animal models of $S$. aureus infection, bound to one another in vitro, in mouse models of $S$. aureus skin infection and bacteraemia and in sera from humans ${ }^{90}$. This binding effectively neutralized the protective effects of these antibodies, providing yet another possible explanation for the failure of antibody-mediated immunity against $S$. aureus in humans ${ }^{90}$. Newer antibody-based vaccines directed against different or multiple components of $S$. aureus, some of which are currently in clinical trials, might be more efficacious and these are reviewed elsewhere $^{91}$. Several of these strategies do not promote antibody-mediated opsonization of the bacteria but instead target $S$. aureus virulence factors, such as a-haemolysin and protein A (using vaccines containing non-toxic forms of the proteins $)^{92,93}$, iron-regulated surface determinant B (which is involved in bacterial iron uptake) ${ }^{94}$ and the $S$. aureus clotting factors coagulase and von Willebrand factor binding protein (which promote fibrin accumulation to wall off an abscess) ${ }^{95}$. However, it is not known how effective an antibody-based vaccine will be against $S$. aureus cutaneous infections as up to one-third of patients suffer recurrent infections ${ }^{96,97}$ despite the fact that many of these patients develop natural antibodies specific for the same components of $S$. aureus that are targeted by these vaccines ${ }^{98}$.

Enhancing IL-1- and IL-17-mediated cutaneous immune responses. The data regarding the role of IL-1 and IL-17 in cutaneous host defence against S. aureus can be combined into one model. This model proposes that IL- 1 functions upstream of the IL-17 response and that both IL-1 and IL-17 can stimulate the expression of pro-inflammatory cytokines, chemokines and adhesion molecules. These molecules induce neutrophil recruitment and the production of antimicrobial peptides to promote immune clearance of an S. aureus cutaneous infection (FIG. 6a). There are several immunomodulation strategies that could be developed, including topically applied preparations or intralesional injections that could work locally in the skin, to enhance IL-1- and IL-17-mediated responses at the site of infection. It should be mentioned that immunomodulation should be used with caution because it has the potential to have harmful effects on patients. Thus, these immunomodulatory therapies would be most relevant for use in certain populations of patients, including patients with congenital or acquired immune defects who are highly susceptible to $S$. aureus skin infections (such as individuals with hyper-IgE syndrome, MYD88 or IRAK4 deficiency or HIV/AIDS) and patients with recurrent infections. In addition, these approaches could be used to treat complicated skin and soft tissue infections (such as cellulitis, abscesses and infected ulcers and wounds) and more invasive infections that are often difficult to treat with antibiotics alone.

Strategies to enhance IL-1-mediated responses might include: activating TLRs using TLR agonists ${ }^{99}$; enhancing inflammasome activation (for example, through the administration of alum ${ }^{100}$, nanoparticles ${ }^{101}$ or ATP $\gamma \mathrm{S}^{102}$ (a form of ATP that is stable in vivo and activates P2X7)); administering recombinant IL-1 $\alpha$ or IL-1 $\beta$ to infected skin $^{26}$; and enhancing IL-1R signalling by administering neutralizing antibodies specific for IL-1RA or the decoy receptor IL-1R2 (REF. 15). Of these strategies, the proof of principle for immunomodulation by TLR activation has been provided by a topical cream formulation of imiquimod, a TLR7 agonist, which is currently used in humans to treat genital warts, actinic keratoses and basal cell carcinomas ${ }^{99}$. Strategies to enhance $\mathrm{T}_{\mathrm{H}} 17$ cell and IL-17-mediated responses could include: administering one or more recombinant cytokines that are known to induce $\mathrm{T}_{\mathrm{H}} 17$ cell responses (such as IL-1 $\alpha$, IL-1 $\beta$, IL-6, IL-21, IL-23 and/or TGF $\beta)^{44,45}$; administering recombinant IL-17A or IL-17F ${ }^{29}$; and administering CCL20 to promote the recruitment of CCR6 ${ }^{+} \mathrm{T}_{\mathrm{H}} 17$ cells $^{86,87}$.

Finally, the end result of IL-1- and IL-17-mediated responses is the promotion of neutrophil recruitment and antimicrobial peptide production. These responses could be enhanced to help combat $S$. aureus cutaneous infections by administering neutrophil-attracting chemokines ${ }^{103}$ or by triggering keratinocyte production of antimicrobial peptides; for example, by administering vitamin D analogues to induce the production of cathelicidins ${ }^{104,105}$. It should be mentioned that any strategy designed to modulate the neutrophilic response will need to maintain neutrophil killing activity (and not simply increase neutrophil number), because $S$. aureus possesses evasion mechanisms that inhibit neutrophil recruitment and function ${ }^{26}$, and these can promote bacterial survival within neutrophils ${ }^{106,107}$. 
a
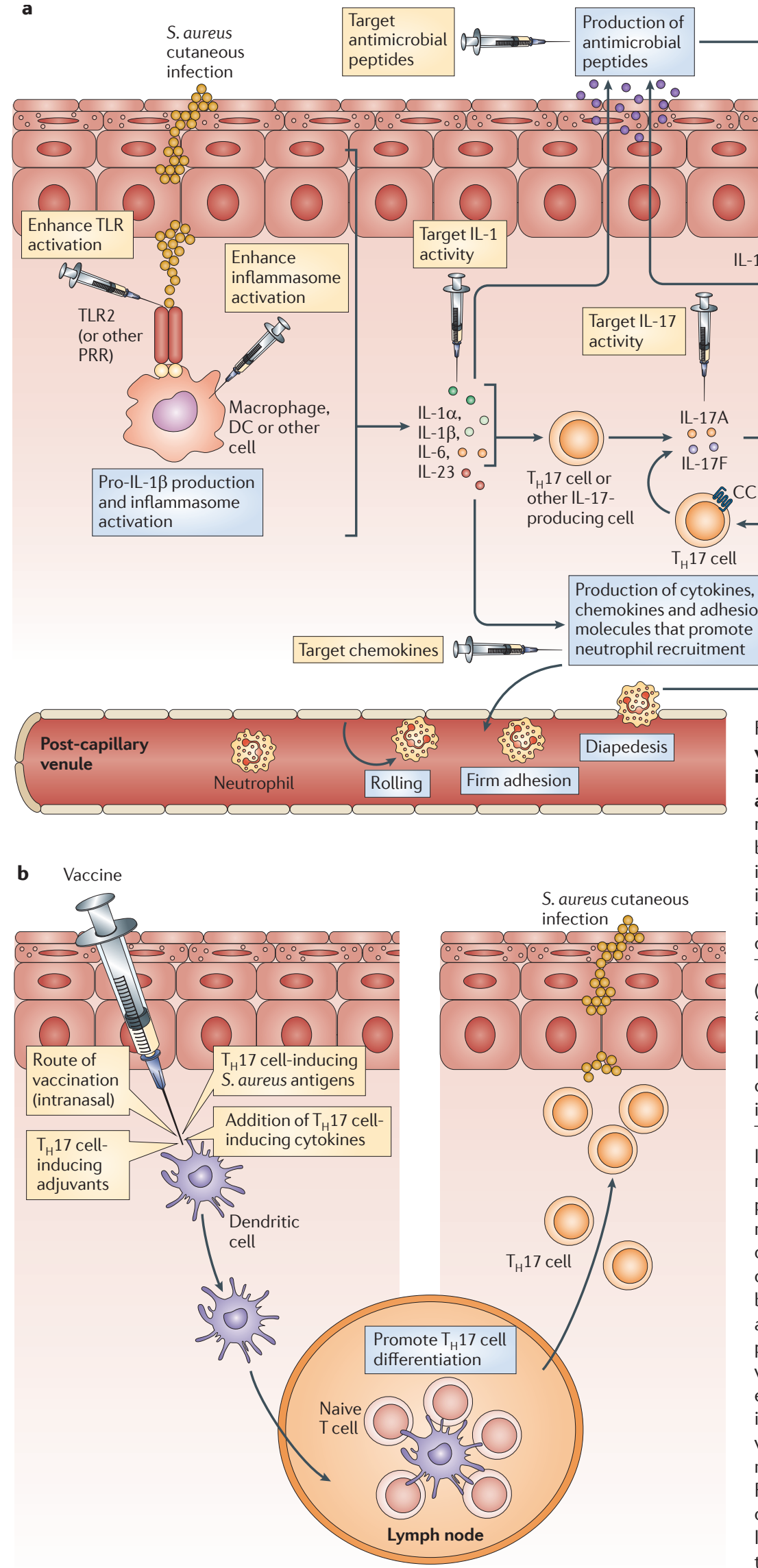

Figure 6 | Potential immunomodulatory and vaccination strategies that might help to promote immunity and provide a therapeutic advantage against cutaneous $S$. aureus infections. a |There are many different targets for immunomodulation (indicated by syringes) that might help to induce protective interleukin-1 (IL-1)- and/or IL-17-mediated cutaneous immune responses against Staphylococcus aureus skin infections. Strategies to promote IL-1-mediated activity could include: activating Toll-like receptors (TLRs) using TLR agonists; enhancing inflammasome activation (for example, using alum, nanoparticles or ATP $\gamma \mathrm{S}$ ); administering recombinant IL-1 $\alpha$ or IL-1 $\beta$; and blocking IL-1 receptor antagonist (IL-1RA) or the decoy receptor IL-1R2. Strategies to promote IL-17-mediated activity could involve the administration of various factors, including: recombinant cytokines that are known to induce Thelper $17\left(\mathrm{~T}_{\mathrm{H}} 17\right)$ cell responses (such as IL-1 $\alpha$, IL-1 $\beta$, IL-6, IL-21, IL-23 and transforming growth factor- $\beta$ (TGF $\beta)$ ); recombinant IL-17A or IL-17F; and CCL20, which would promote the additional recruitment of CC-chemokine receptor $6(C C R 6)^{+} T_{H} 17$ cells. Neutrophil recruitment could be enhanced by administering neutrophil-attracting chemokines. Finally, antimicrobial peptide production by keratinocytes could be enhanced (for example, by administering vitamin $D$ analogues to induce the production of cathelicidins). $\mathbf{b}$ |The figure shows potential vaccination strategies aimed at preferentially producing an effective $T_{H} 17$ cell response against cutaneous $S$. aureus infections. These include using an intranasal route of vaccination, using adjuvants that promote $T_{H} 17$ cell responses (such as cholera toxin, curdlan and complete Freund's adjuvant), addition of recombinant $T_{H} 17$ cell-inducing cytokines during vaccination (such as IL-1 $\beta$, IL-6, IL-21, IL-23 and TGF $\beta$ ) and vaccination with antigens that preferentially induce $T_{H} 17$ cell responses against S. aureus. DC, dendritic cell. 
Potential vaccination strategies to promote $T_{H} 17$ cells. Vaccination strategies that promote the homing of $\mathrm{T}_{\mathrm{H}} 17$ cells to the skin might be an effective way to prevent $S$. aureus cutaneous infections. Vaccines that induce $\mathrm{T}_{\mathrm{H}} 17$ cell responses have been effective in mouse models against various pathogens ${ }^{108}$. In addition, two $\mathrm{T}_{\mathrm{H}} 17$ cellinducing vaccines - that incorporated either a recombinant amino-terminal region of the candidal adhesion protein Als3 (rAls3p-N) or the fibrinogen domain of $S$. aureus clumping factor A (both in the presence of alum) - protected mice against an intravenous $S$. aureus challenge $\mathrm{e}^{109,110}$. These studies indicate that a $\mathrm{T}_{\mathrm{H}} 17$ cellinducing vaccine could potentially be effective against S. aureus cutaneous infections. Several strategies have been shown to mainly induce $\mathrm{T}_{\mathrm{H}} 17$ cell responses. These approaches include: using an intranasal route of vaccination ${ }^{111}$; using adjuvants that promote $\mathrm{T}_{\mathrm{H}} 17$ cell responses (such as cholera toxin, curdlan and complete Freund's adjuvant ${ }^{108}$ ); adding recombinant $\mathrm{T}_{\mathrm{H}} 17$ cell-inducing cytokines during vaccination (such as IL-1 $\beta$, IL-6, IL-21, IL-23 and TGF $\beta)^{44,45}$; and incorporating antigens that predominantly induce $\mathrm{T}_{\mathrm{H}} 17$ cell responses against $S$. aureus. A large scale proteomic screen of an expression library of antigens that primarily induce $\mathrm{T}_{\mathrm{H}} 17$ cell cytokines has been proposed as a strategy for future vaccine development against $S$. pneumoniae ${ }^{112}$, and a similar strategy might be effective for the development of a $\mathrm{T}_{\mathrm{H}} 17$ cell-inducing $S$. aureus vaccine. One particular challenge of such a vaccine is that the $\mathrm{T}_{\mathrm{H}} 17$ cell response must be specific for $S$. aureus to avoid any off-target effects, in particular because $\mathrm{T}_{\mathrm{H}} 17$ cells can contribute to promoting autoimmunity, as seen in the inflammatory skin disease psoriasis ${ }^{44,45}$.

\section{Conclusions and perspectives}

To develop an effective immunotherapy or vaccine against $S$. aureus infection, it is important to understand the protective cutaneous immune responses against $S$. aureus, as the vast majority of $S$. aureus and MRSA infections occur in the skin ${ }^{1,2}$. As with other extracellular pathogens, initial vaccination strategies against $S$. aureus were designed to promote antibody-mediated opsonization of the bacteria and subsequent phagocytosis and bacterial clearance ${ }^{88,89}$. Newer antibody-based vaccines, which are directed against $S$. aureus virulence factors, have had promising results in animal models ${ }^{91}$. However, it remains unclear whether these antibody-based strategies alone will offer protection against $S$. aureus infections in humans given the failure of previous antibody-based vaccines in clinical trials ${ }^{88,89}$ and the fact that many patients already have natural antibodies specific for the same components that are targeted in these vaccines ${ }^{98}$.

It has been known for some time that patients with defects in neutrophil number or function have an increased susceptibility to $S$. aureus infections ${ }^{53}$. However, no immunotherapies have been specifically designed to directly promote the neutrophilic response against $S$. aureus infections. In this Review, we describe the recent findings that have identified specific immune impairments in patients with genetic diseases who are predisposed to $S$. aureus infections at cutaneous sites. These include patients with defective IL-1R and/or TLR signalling (as a result of MYD88 or IRAK4 deficiency $)^{17-20}$ and impaired $\mathrm{T}_{\mathrm{H}} 17$ cell responses (as a result of hyper-IgE syndrome $)^{21-24}$. Mouse models of S. aureus cutaneous infection have supported these findings and further elucidated that IL-1 (REFS 25-27) and IL-17 (REFS 28,29) are essential for promoting neutrophil recruitment to the skin. Thus, these studies have directly linked the adaptive $\mathrm{T}_{\mathrm{H}} 17$ cell-mediated immune response with the protective neutrophilic response against $S$. aureus infections in the skin. These insights provide a new rationale for future research directed at targeting IL-1- and IL-17-mediated responses through immunomodulation or vaccination. In particular, the generation of a vaccine that would induce $S$. aureusspecific $\mathrm{T}_{\mathrm{H}} 17$ cells at cutaneous sites of infection might be a particularly attractive strategy to promote protection. If successful, these strategies could be an alternative approach for future immunotherapy to combat $S$. aureus and MRSA infections in the skin and perhaps at other epithelial sites of infection.
1. McCaig, L. F., McDonald, L. C., Mandal, S. \& Jernigan, D. B. Staphylococcus aureus-associated skin and soft tissue infections in ambulatory care Emerg. Infect. Dis. 12, 1715-1723 (2006).

2. Moran, G. J. et al. Methicillin-resistant S. aureus infections among patients in the emergency department. N. Engl. J. Med. 355, 666-674 (2006) Lowy, F. D. Staphylococcus aureus infections. N. Engl. J. Med. 339, 520-532 (1998).

4. Daum, R. S. Clinical practice. Skin and soft-tissue infections caused by methicillin-resistant Staphylococcus aureus. N. Engl. J. Med. 357, 380-390 (2007).

5. Deleo, F. R., Otto, M., Kreiswirth, B. N. \& Chambers, H. F. Community-associated meticillinresistant Staphylococcus aureus. Lancet 375 1557-1568 (2010).

6. Klevens, R. M. et al. Invasive methicillin-resistant Staphylococcus aureus infections in the United States. JAMA 298, 1763-1771 (2007).

7. Boucher, H. W. \& Corey, G. R. Epidemiology of methicillin-resistant Staphylococcus aureus. Clin. Infect. Dis. 46, S344-S349 (2008).

8. Song, J. H. et al. Spread of methicillin-resistant Staphylococcus aureus between the community and the hospitals in Asian countries: an ANSORP study. J. Antimicrob. Chemother. 66, 1061-1069 (2011).
9. Niniou, I. et al. Clinical and molecular epidemiology of community-acquired, methicillin-resistant Staphylococcus aureus infections in children in central Greece. Eur. J. Clin. Microbiol. Infect. Dis. 27, 831-837 (2008)

10. Elston, J. W. \& Barlow, G. D. Community-associated MRSA in the United Kingdom. J. Infect. 59, 149-155 (2009).

11. Lorette, G., Beaulieu, P., Allaert, F. A., Mahmoudi, A \& Jarlier, V. Superficial community-acquired skin infections: prevalence of bacteria and antibiotic susceptibility in France. J. Eur. Acad. Dermatol. Venereol. 23, 1423-1426 (2009).

12. Miller, L. G. \& Diep, B. A. Clinical practice: colonization, fomites, and virulence: rethinking the pathogenesis of community-associated methicillinresistant Staphylococcus aureus infection. Clin. Infect. Dis. 46, 752-760 (2008).

13. Dryden, M. S. Complicated skin and soft tissue infection J. Antimicrob. Chemother. 65, iii35-iii44 (2010).

14. Moellering, R. C. Jr. The problem of complicated skin and skin structure infections: the need for new agents. J. Antimicrob. Chemother. 65, iv3-iv8 (2010).

15. Kupper, T. S. \& Fuhlbrigge, R. C. Immune surveillance in the skin: mechanisms and clinical consequences. Nature Rev. Immunol. 4, 211-222 (2004).
16. Nestle, F. O., Di, M. P., Qin, J. Z. \& Nickoloff, B. J. Skin immune sentinels in health and disease. Nature Rev. Immunol. 9, 679-691 (2009). References 15 and 16 are excellent reviews of cutaneous immune responses.

17. Ku, C. L. et al. Selective predisposition to bacterial infections in IRAK-4-deficient children: IRAK-4-dependent TLRs are otherwise redundant in protective immunity. J. Exp. Med. 204, 2407-2422 (2007).

18. Picard, C. et al. Pyogenic bacterial infections in humans with IRAK-4 deficiency. Science 299 2076-2079 (2003)

19. von Bernuth, H. et al. Pyogenic bacterial infections in humans with MyD88 deficiency. Science 321, 691-696 (2008). References 18 and 19 found that humans deficient in MYD88 or IRAK4 have an increased susceptibility to a narrow range of pyogenic bacterial infections, including $S$. aureus skin infections, which indicates that IL-1R and TLR family members are crucial for host defence against these infections.

20. Picard, C. et al. Clinical features and outcome of patients with IRAK- 4 and MyD88 deficiency. Medicine 89, 403-425 (2010). 
21. Milner, J. D. et al. Impaired T 17 cell differentiation in subjects with autosomal dominant hyper-IgE syndrome. Nature 452, 773-776 (2008)

22. Ma, C. S. et al. Deficiency of Th17 cells in hyper IgE syndrome due to mutations in STAT3. J. Exp. Med. 205, 1551-1557 (2008)

23. Renner, E. D. et al. Novel signal transducer and activator of transcription 3 (STAT3) mutations, reduced $T_{H} 17$ cell numbers, and variably defective STAT3 phosphorylation in hyper-lgE syndrome. J. Allergy Clin. Immunol. 122, 181-187 (2008). References 21-23 were the first to show that patients with autosomal dominant hyper-IgE syndrome have STAT3 mutations that render them deficient in $T_{H} 17$ cells; this indicates that $T_{H} 17$ cell responses are crucial for immunity against $S$. aureus skin infections.

24. Al, K. S. et al. Defects along the $T_{H} 17$ differentiation pathway underlie genetically distinct forms of the hyper IgE syndrome. J. Allergy Clin. Immunol. 124 342-348 (2009)

25. Miller, L. S. et al. MyD88 mediates neutrophil recruitment initiated by IL-1R but not TLR2 activation in immunity against Staphylococcus aureus. Immunity 24, 79-91 (2006).

This study showed that although IL-1R and TLR2 both signal through the adaptor molecule MYD88, IL-1 R-MYD88 signalling is a more important determinant than TLR2-MYD88 signalling for neutrophil recruitment and host defence against $S$. aureus cutaneous infection in mice.

26. Miller, L. S. et al. Inflammasome-mediated production of IL-1 $\beta$ is required for neutrophil recruitment against Staphylococcus aureus in vivo. J. Immunol. 179 6933-6942 (2007).

27. Munoz-Planillo, R., Franchi, L., Miller, L. S. \& Nunez, C. A critical role for hemolysins and bacterial lipoproteins in Staphylococcus aureus-induced activation of the Nlrp3 inflammasome. J. Immunol. 183, 3942-3948 (2009).

28. Ishigame, H. et al. Differential roles of interleukin-17A and $-17 \mathrm{~F}$ in host defense against mucoepithelial bacterial infection and allergic responses. Immunity 30, 108-119 (2009).

This study found that mice deficient in both IL-17A and IL-17F developed spontaneous $S$. aureus skin infections but had no impairment in immunity against an intravenous $S$. aureus challenge, which indicates that IL-17-mediated responses are essential for immunity at cutaneous sites of infection.

29. Cho, J. S. et al. IL-17 is essential for host defense against cutaneous Staphylococcus aureus infection in mice. J. Clin. Invest. 120, 1762-1773 (2010). This study identified an important role for IL-17-producing $\gamma \delta$ T cells in mouse skin. These cells promoted neutrophil recruitment and host defence against a cutaneous infection with S. aureus.

30. Fournier B \& Philpott D J Recognition of Staphylococcus aureus by the innate immune system. Clin. Microbiol. Rev. 18, 521-540 (2005).

31. Otto, M. Basis of virulence in community-associated methicillin-resistant Staphylococcus aureus. Annu. Rev. Microbiol. 64, 143-162 (2010).

32. Foster, T. J. Immune evasion by staphylococci. Nature Rev. Microbiol. 3, 948-958 (2005)

33. Grice, E. A. \& Segre, J. A. The skin microbiome Nature Rev. Microbiol. 9, 244-253 (2011).

34. Grice, E. A. et al. Topographical and temporal diversity of the human skin microbiome. Science 324, 1190-1192 (2009)

35. Schauber, J. \& Gallo, R. L. Antimicrobial peptides and the skin immune defense system. J. Allergy Clin. Immunol. 124, R13-R18 (2009).

36. Otto, M. Staphylococcus colonization of the skin and antimicrobial peptides. Expert Rev. Dermatol. 5, 183-195 (2010)

37. Kawai, T. \& Akira, S. The role of pattern-recognition receptors in innate immunity: update on Toll-like receptors. Nature Immunol. 11, 373-384 (2010).

38. Molne, L., Verdrengh, M. \& Tarkowski, A. Role of neutrophil leukocytes in cutaneous infection caused by Staphylococcus aureus. Infect. Immun. $\mathbf{6 8}$ 6162-6167 (2000)

39. Ley, K., Laudanna, C., Cybulsky, M. I. \& Nourshargh, S. Getting to the site of inflammation: the leukocyte adhesion cascade updated. Nature Rev. Immunol. 7, 678-689 (2007).

40. Kim, M. H. et al. Neutrophil survival and c-kit ${ }^{+}$ progenitor proliferation in Staphylococcus aureus-infected skin wounds promote resolution. Blood 117, 3343-3352 (2011)

41. Kobayashi, S. D. \& Deleo, F. R. Role of neutrophils in innate immunity: a systems biology-level approach. Wiley Interdiscip. Rev. Syst. Biol. Med. 1, 309-333 (2009)

42. Segal, A. W. How neutrophils kill microbes. Annu. Rev. Immunol. 23, 197-223 (2005)

43. Corbin, B. D. et al. Metal chelation and inhibition of bacterial growth in tissue abscesses. Science 319, 962-965 (2008)

44. Cua, D. J. \& Tato, C. M. Innate IL-17-producing cells the sentinels of the immune system. Nature Rev. Immunol. 10, 479-489 (2010).

45. Korn, T., Bettelli, E., Oukka, M. \& Kuchroo, V. K. IL-17 and Th1 7 cells. Annu. Rev. Immunol. 27 485-517 (2009)

46. Duhen, T., Geiger, R., Jarrossay, D., Lanzavecchia, A \& Sallusto, F. Production of interleukin 22 but not interleukin 17 by a subset of human skin-homing memory T cells. Nature Immunol. 10, 857-863 (2009)

47. Eyerich, S. et al. Th22 cells represent a distinct human T cell subset involved in epidermal immunity and remodeling. J. Clin. Invest. 119, 3573-3585 (2009).

48. Zheng, Y. et al. Interleukin-22, a $\mathrm{T}_{4} 17$ cytokine, mediates IL-23-induced dermal inflammation and acanthosis. Nature 445, 648-651 (2007).

49. Peric, M. et al. IL-17A enhances vitamin D3-induced expression of cathelicidin antimicrobial peptide in human keratinocytes. J. Immunol. 181, 8504-8512 (2008)

50. Liang, S. C. et al. Interleukin (IL)-22 and IL-17 are coexpressed by Th 17 cells and cooperatively enhance expression of antimicrobial peptides. J. Exp. Med. 203, 2271-2279 (2006).

51. Caruso, R. et al. Involvement of interleukin-21 in the epidermal hyperplasia of psoriasis. Nature Med. 15, 1013-1015 (2009)

52. Gonzalez-Barca, E., Carratala, J., Mykietiuk, A. Fernandez-Sevilla, A. \& Gudiol, F. Predisposing factors and outcome of Staphylococcus aureus bacteremia in neutropenic patients with cancer. Eur. J. Clin. Microbiol. Infect. Dis. 20, 117-119 (2001).

53. Bouma, G. Ancliff, P. J., Thrasher, A. J. \& Burns, S. O. Recent advances in the understanding of genetic defects of neutrophil number and function. Br. J. Haematol. 151, 312-326 (2010).

54. Lakshman, R. \& Finn, A. Neutrophil disorders and their management. J. Clin. Pathol. 54, 7-19 (2001).

55. Andrews, T. \& Sullivan, K. E. Infections in patients with inherited defects in phagocytic function. Clin. Microbiol. Rev. 16, 597-621 (2003).

56. Alba-Loureiro, T. C. et al. Neutrophil function and metabolism in individuals with diabetes mellitus. Braz. J. Med. Biol. Res. 40, 1037-1044 (2007)

57. Chonchol, M. Neutrophil dysfunction and infection risk in end-stage renal disease. Semin. Dial. 19, 291-296 (2006).

58. Casanova, J. L., Abel, L. \& Quintana-Murci, L. Human TLRs and IL-1 Rs in host defense: natural insights from evolutionary, epidemiological, and clinical genetics. Annu. Rev. Immunol. 29, 447-491 (2011).

59. Minegishi, Y et al. Dominant-negative mutations in the DNA-binding domain of STAT3 cause hyper-IgE syndrome. Nature 448, 1058-1062 (2007).

60. Holland, S. M. et al. STAT3 mutations in the hyper-IgE syndrome. N. Engl. J. Med. 357 1608-1619 (2007)

61. Engelhardt, K. R. et al. Large deletions and point mutations involving the dedicator of cytokinesis 8 (DOCK8) in the autosomal-recessive form of hyper-lgE syndrome. J. Allergy Clin. Immunol. 124, 1289-1302 (2009).

62. Zhang, Q. et al. Combined immunodeficiency associated with DOCK8 mutations. N. Engl. J. Med. 361, 2046-2055 (2009).

63. Grivennikov, S. I. \& Karin, M. Dangerous liaisons: STAT3 and NF-kB collaboration and crosstalk in cancer. Cytokine Growth Factor Rev. 21, 11-19 (2010).

64. Guttman-Yassky, E. et al. Low expression of the IL-23/ Th17 pathway in atopic dermatitis compared to psoriasis. J. Immunol. 181, 7420-7427 (2008).

65. Ong, P. Y. et al. Endogenous antimicrobial peptides and skin infections in atopic dermatitis. N. Engl. J. Med. 347, 1151-1160 (2002)

66. Nograles, K. E. et al. IL-22-producing “T22” T cells account for upregulated IL-22 in atopic dermatitis despite reduced IL-17-producing $\mathrm{T}_{4} 17 \mathrm{~T}$ cells. J. Allergy Clin. Immunol. 123, 1244-1252 (2009).
67. Manfredi, R., Calza, L. \& Chiodo, F. Epidemiology and microbiology of cellulitis and bacterial soft tissue infection during HIV disease: a 10-year survey. J. Cutan. Pathol. 29, 168-172 (2002).

68. Prendergast, A. et al. HIV-1 infection is characterized by profound depletion of CD $161^{+}$Th 17 cells and gradual decline in regulatory T cells. AIDS $\mathbf{2 4}$, 491-502 (2010)

69. Kisand, K. et al. Chronic mucocutaneous candidiasis in APECED or thymoma patients correlates with autoimmunity to Th17-associated cytokines. J. Exp. Med. 207, 299-308 (2010)

70. Puel, A. et al. Autoantibodies against IL-17A, IL-17F, and IL-22 in patients with chronic mucocutaneous candidiasis and autoimmune polyendocrine syndrome type I. J. Exp. Med. 207, 291-297 (2010).

71. Puel, A. et al. Chronic mucocutaneous candidiasis in humans with inborn errors of interleukin-17 immunity. Science 332, 65-68 (2011).

References 69-71 showed that patients with chronic mucocutaneous candidiasis have mutations of or autoantibodies specific for components of the $T_{H} 17$ cell pathway, directly implicating $T_{H} 17$ cell responses in immunity against mucocutaneous candidal infections and $S$. aureus skin infections.

72. Gaffen, S. L. Structure and signalling in the IL-17 receptor family. Nature Rev. Immunol. 9, 556-567 (2009).

73. Salliot, C., Dougados, M. \& Gossec, L. Risk of serious infections during rituximab, abatacept and anakinra treatments for rheumatoid arthritis: meta-analyses of randomised placebo-controlled trials. Ann. Rheum. Dis. 68, 25-32 (2009).

74. Cho, J. S. et al. Noninvasive in vivo imaging to evaluate immune responses and antimicrobial therapy against Staphylococcus aureus and USA300 MRSA skin infections. J. Invest. Dermatol. 131, 907-915 (2011).

75. Olaru, F. \& Jensen, L. E. Staphylococcus aureus stimulates neutrophil targeting chemokine expression in keratinocytes through an autocrine IL-1 a signaling loop. J. Invest. Dermatol. 130, 1866-1876 (2010)

76. Schroder, K. \& Tschopp, J. The inflammasomes. Cell 140, 821-832 (2010).

77. Hruz, P. et al. NOD2 contributes to cutaneous defense against Staphylococcus aureus through a-toxin-dependent innate immune activation. Proc. Natl Acad. Sci. USA 106, 12873-12878 (2009). This is an important paper showing that NOD2, an intracellular PRR that recognizes the peptidoglycan breakdown product muramyl dipeptide, contributes to host defence against cutaneous infection with $S$. aureus in mice by inducing the production of IL-1 $\beta$, which induces IL-6-mediated bacterial killing by neutrophils.

78. Mariathasan, S. et al. Cryopyrin activates the inflammasome in response to toxins and ATP. Nature 440, 228-232 (2006).

79. Franchi, L., Kanneganti, T. D., Dubyak, G. R. \& Nunez, G. Differential requirement of $\mathrm{P} 2 \mathrm{X} 7$ receptor and intracellular $\mathrm{K}^{+}$for caspase- 1 activation induced by intracellular and extracellular bacteria. J. Biol. Chem. 282, 18810-18818 (2007).

80. Craven, R. R. et al. Staphylococcus aureus a-hemolysin activates the NLRP3-inflammasome in human and mouse monocytic cells. PLOS ONE 4, e7446 (2009)

81. Shimada, T. et al. Staphylococcus aureus evades lysozyme-based peptidoglycan digestion that links phagocytosis, inflammasome activation, and IL-1 $\beta$ secretion. Cell Host Microbe 7, 38-49 (2010).

82. Minegishi, Y. et al. Molecular explanation for the contradiction between systemic Th 17 defect and localized bacterial infection in hyper-IgE syndrome. J. Exp. Med. 206, 1291-1301 (2009). This study found that human keratinocytes and bronchial epithelial cells have a much stronger dependence on $\mathrm{T}_{H} 17$ cell cytokines than other cell types, providing an explanation for the predisposition of patients with hyper-lgE syndrome to $S$. aureus infections in the skin and lung and not at other sites.

83. Clark, R. A. et al. The vast majority of $\mathrm{CLA}^{+} \mathrm{T}$ cells are resident in normal skin. J. Immunol. 176 4431-4439 (2006).

84. Gray, E. E., Suzuki, K. \& Cyster, J. G. Cutting edge: identification of a motile IL-17-producing $\gamma \delta \mathrm{T}$ cell population in the dermis. J. Immunol. 186 6091-6095 (2011). 
85. Sumaria, N. et al. Cutaneous immunosurveillance by self-renewing dermal $\gamma \delta$ T cells. J. Exp. Med. 208 505-518 (2011)

86. Harper, E. G. et al. Th17 cytokines stimulate CCL20 expression in keratinocytes in vitro and in vivo: implications for psoriasis pathogenesis. J. Invest Dermatol. 129, 2175-2183 (2009).

87. Hedrick, M. N. et al. CCR6 is required for IL-23-induced psoriasis-like inflammation in mice. J. Clin. Invest. 119, 2317-2329 (2009).

88. Deresinski, S. \& Herrera, V. Immunotherapies for Staphylococcus aureus: current challenges and future prospects. Infect. Control Hosp. Epidemiol. 31, S45-S47 (2010)

89 Schaffer, A. C. \& Lee, J. C. Vaccination and passive immunisation against Staphylococcus aureus. Int J. Antimicrob. Agents 32, S71-S78 (2008).

90. Skurnik, D. et al. Animal and human antibodies to distinct Staphylococcus aureus antigens mutually neutralize opsonic killing and protection in mice. J. Clin. Invest. 120, 3220-3233 (2010).

91. Kobayashi, S. D. \& Deleo, F. R. A MRSA-terious enemy among us: boosting MRSA vaccines. Nature Med. 17, 168-169 (2011).

92. Kennedy, A. D. et al. Targeting of $\alpha$-hemolysin by active or passive immunization decreases severity of USA300 skin infection in a mouse model. J. Infect. Dis. 202, 1050-1058 (2010).

93. Kim, H. K., Cheng, A. G., Kim, H. Y., Missiakas, D. M $\&$ Schneewind, O. Nontoxigenic protein A vaccine for methicillin-resistant Staphylococcus aureus infections in mice. J. Exp. Med. 207, 1863-1870 (2010).

94. Harro, C. et al. Safety and immunogenicity of a novel Staphylococcus aureus vaccine: results from the first study of the vaccine dose range in humans. Clin. Vaccine Immunol. 17, 1868-1874 (2010).

95. Cheng, A. G. et al. Contribution of coagulases towards Staphylococcus aureus disease and protective immunity. PLoS Pathog. 6, e1001036 (2010).

96. Miller, L. G. et al. A prospective investigation of outcomes after hospital discharge for endemic, community-acquired methicillin-resistant and -susceptible Staphylococcus aureus skin infection. Clin. Infect. Dis. 44, 483-492 (2007).

97. Huang, S. S. \& Platt, R. Risk of methicillin-resistant Staphylococcus aureus infection after previous infection or colonization. Clin. Infect. Dis. 36 281-285 (2003).

98. Holtfreter, S., Kolata, J. \& Broker, B. M. Towards the immune proteome of Staphylococcus aureus the anti-S. aureus antibody response. Int. J. Med. Microbiol. 300, 176-192 (2010).

99. Ulevitch, R. J. Therapeutics targeting the innate immune system. Nature Rev. Immunol. 4, 512-520 (2004)

100. Eisenbarth, S. C., Colegio, O. R., O'Connor, W., Sutterwala, F. S. \& Flavell, R. A. Crucial role for the Nalp3 inflammasome in the immunostimulatory properties of aluminium adjuvants. Nature $\mathbf{4 5 3}$ 1122-1126 (2008)

101. Yazdi, A. S. et al. Nanoparticles activate the NLR pyrin domain containing 3 (NIrp3) inflammasome and cause pulmonary inflammation through release of IL- $1 \alpha$ and IL-1 $\beta$. Proc. Natl Acad. Sci. USA 107, 19449-19454 (2010).

102. Granstein, R. D. et al. Augmentation of cutaneous immune responses by ATP $\gamma$ S: purinergic agonists define a novel class of immunologic adjuvants. J. Immunol. 174, 7725-7731 (2005).

103. Shahangian, A. et al. Type I IFNs mediate development of postinfluenza bacterial pneumonia in mice. J. Clin. Invest. 119, 1910-1920 (2009).

104. Liu, P. T. et al. Toll-like receptor triggering of a vitamin D-mediated human antimicrobial response. Science 311, 1770-1773 (2006).

105. Schauber, J. et al. Injury enhances TLR2 function and antimicrobial peptide expression through a vitamin D-dependent mechanism. J. Clin. Invest. 117, 803-811 (2007)

106. McLoughlin, R. M., Lee, J. C., Kasper, D. L. $\&$ Tzianabos, A. O. IFN- $\gamma$ regulated chemokine production determines the outcome of Staphylococcus aureus infection. J. Immunol. 181, 1323-1332 (2008).

107. Palazzolo-Ballance, A. M. et al. Neutrophil microbicides induce a pathogen survival response in community- associated methicillin-resistant Staphylococcus aureus. J. Immunol. 180, 500-509 (2008).

108. Lin, Y., Slight, S. R. \& Khader, S. A. Th 17 cytokines and vaccine-induced immunity. Semin. Immunopathol. 32, 79-90 (2010).

109. Lin, L. et al. Th1-Th17 cells mediate protective adaptive immunity against Staphylococcus aureus and Candida albicans infection in mice. PLoS Pathog. 5, e1000703 (2009)

This paper showed that a vaccination strategy that provided protection against intravenous challenge with $S$. aureus depended on the generation of $\mathrm{T}_{\mathrm{H}} 17$ cells, which promoted neutrophil recruitmen and more effective bacterial clearance.

110. Narita, K. et al. Role of interleukin-17A in cellmediated protection against Staphylococcus aureus infection in mice immunized with the fibrinogenbinding domain of clumping factor A. Infect. Immun 78, 4234-4242 (2010)

111. Zygmunt, B. M., Rharbaoui, F., Groebe, L. \& Guzman, C. A. Intranasal immunization promotes Th1 7 immune responses. J. Immunol. 183, 6933-6938 (2009).

112. Moffitt, K. L. et al. $\mathrm{T}_{H} 17$-based vaccine design for prevention of Streptococcus pneumoniae colonization. Cell Host Microbe 9, 158-165 (2011).

\section{Acknowledgements}

L.S.M. is supported by grants from the US National Institutes of Health (R01 Al078910 and R03 AR054534). J.S.C. is supported by the Dermatology Scientist Training Program at UCLA from the US National Institutes of Health (T32 AR058921)

\section{Competing interests statement}

The authors declare competing financial interests: see web version for details.

FURTHER INFORMATION

Lloyd S. Miller's homepage: http://www.derm.med.ucla.edu/

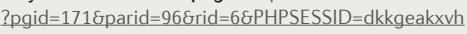

ALL LINKS ARE ACTIVE IN THE ONLINE PDF 